\title{
A posteriori error estimates for the effective Hamiltonian of dislocation dynamics
}

\author{
S. Cacace*, A. Chambolle ${ }^{\dagger}$, R. Monneau*
}

\begin{abstract}
We study an implicit and discontinuous scheme for a non-local Hamilton-Jacobi equation modelling dislocation dynamics. For the evolution problem, we prove an a posteriori estimate of Crandall-Lions type for the error between continuous and discrete solutions. We deduce an a posteriori error estimate for the effective Hamiltonian associated to a stationary cell problem. In dimension one and under suitable assumptions, we also give improved a posteriori estimates. Numerical simulations are provided.
\end{abstract}

AMS Classification: 35B10, 35B27, 35F20, 35F25, 35Q72, 49L25, 65M06, 65M15

Keywords: dislocation dynamics, Hamilton-Jacobi, implicit scheme, a posteriori error estimate, effective Hamiltonian, viscosity solutions.

\section{Introduction}

In this paper, we are interested in a first order non-local Hamilton-Jacobi equation describing dislocation dynamics. For general references on dislocation theory, we refer for instance the reader to [13] and the book [18]. The model studied in the present paper has been introduced in [15] where the periodic homogenization of such equation has been obtained. To this end an effective Hamiltonian has been defined and was interpreted as a visco-plastic law in mechanics (see also [19] for a section on the mechanical interpretation). Therefore the numerical computation of this effective Hamiltonian is of particular importance and is the main subject of the present work. In this paper, we will define a numerical scheme for which we will show a posteriori error estimates on the effective Hamiltonian. These a posteriori error estimates will be illustrated by numerical simulations.

\subsection{The continuous cell problem}

To be precise, for any $p \in \mathbb{R}^{N}$ and any function $u: \mathbb{R}^{N} \rightarrow \mathbb{R}$ satisfying

$$
x \mapsto u(x)-p \cdot x \text { is bounded and } \mathbb{Z}^{N} \text {-periodic },
$$

we consider the following non-local operator:

$$
M_{p}[u](x):=\int_{\mathbb{R}^{N}} \mathcal{J}(z)\{E(u(x+z)-u(x))-p \cdot z\} d z,
$$

which describes the force acting on dislocations, represented by the level sets of the function $u$. Here the function $E: \mathbb{R} \rightarrow \mathbb{R}$ is the following odd modification of the integer part:

$$
E(\alpha)=\left\{\begin{array}{lll}
k & \text { if } \quad \alpha=k \in \mathbb{Z} \\
k+\frac{1}{2} & \text { if } \quad k<\alpha<k+1 \quad \text { with } \quad k \in \mathbb{Z}
\end{array}\right.
$$

\footnotetext{
${ }^{*}$ CERMICS, Ecole nationale des Ponts et Chaussées, 6 et 8 avenue Blaise Pascal, Cité Descartes, Champs-sur-Marne, 77455 Marne-la-Vallée Cedex 2, France

${ }^{\dagger}$ CMAP, Ecole Polytechnique CNRS, Route de Saclay, 91128 Palaiseau Cedex, France
} 
and the kernel $\mathcal{J}$ satisfies

$$
\left\{\begin{array}{l}
\mathcal{J} \in W^{1,1}\left(\mathbb{R}^{N}\right), \quad \text { and } \quad \mathcal{J}(-z)=\mathcal{J}(z) \geq 0 \text { for all } z \in \mathbb{R}^{N} \\
\exists R_{0}>0 \text { and } \exists g \in C^{0}\left(\mathbf{S}^{N-1}\right), \text { such that } \mathcal{J}(z)=\frac{g(z /|z|)}{|z|^{N+1}}>0 \text { for }|z| \geq R_{0} .
\end{array}\right.
$$

The fact that the kernel is nonnegative is essential to recover good properties of the non-local operator $M_{p}$ and to finally work in the framework of viscosity solutions (see [20] for a viscosity framework with more general kernels). The second line of (1.3) precises the behaviour of the kernel whose slow decay at infinity is related to the long range interactions between dislocations. The function $g$ allows to consider anisotropic kernels.

We also introduce a periodic function $c_{0}: \mathbb{R}^{N} \rightarrow \mathbb{R}$ which satisfies precisely

$$
c_{0} \in W^{1, \infty}\left(\mathbb{R}^{N}\right) \quad \text { and } \quad c_{0}(x+k)=c_{0}(x) \quad \text { for all } \quad x \in \mathbb{R}^{N}, \quad k \in \mathbb{Z}^{N}
$$

and define the non-local velocity by

$$
c[u]=c_{0}+M_{p}[u]
$$

Then we consider the following continuous cell problem:

$$
\lambda=|\nabla u| c[u] \quad \text { on } \quad \mathbb{R}^{N},
$$

where $\lambda \in \mathbb{R}$ is a constant which is called the effective Hamiltonian. Equation (1.6) has to be understood in a viscosity sense that will be precised in Section 2.1. Then we have the following result which is a corollary of [15] (see later Section 3.1).

\section{Theorem 1.1 (Existence and uniqueness of $\lambda$ )}

Under assumptions (1.3)-(1.4), for any $p \in \mathbb{R}^{N}$, there exists a unique $\lambda \in \mathbb{R}$ such that there exists a function $u$ satisfying (1.1) which is a viscosity solution of (1.6).

Recall that in general there is no uniqueness of the solution $u$ and also that $u$ could be discontinuous.

\subsection{The discrete cell problem}

We now introduce a discretization step in space $\Delta x>0$ with $\frac{1}{\Delta x} \in \mathbb{N}$ and nodes $x_{I}=\left(i_{1} \Delta x, \ldots, i_{N} \Delta x\right)$ for $I=\left(i_{1}, \ldots, i_{N}\right) \in \mathbb{Z}^{N}$. We assume that

$$
u\left(x_{I}\right) \text { is approximated by } v_{I},
$$

where the function $v: \mathbb{Z}^{N} \rightarrow \mathbb{R}$ satisfies

$$
I \mapsto v_{I}-p \cdot x_{I} \text { is bounded and }\left(\frac{1}{\Delta x} \mathbb{Z}\right)^{N} \text {-periodic . }
$$

We then introduce a scheme such that

$$
(|\nabla u| c[u])\left(x_{I}\right) \quad \text { is approximated by } \quad R_{I}[v] .
$$

This scheme is presented in details in Section 2.2 and is strongly inspired from [14]. Then the discrete cell problem is the following

$$
\lambda^{d}=R[v] \quad \text { on } \quad \mathbb{Z}^{N}
$$

where $\lambda^{d} \in \mathbb{R}$ is a constant (the superscript $d$ beeing here to recall that this is the discrete problem). Again, equation (1.8) has to be understood in the sense of viscosity solution for the scheme (see Definition 2.2). Because of the discontinuity of the scheme, related to the discontinuity of the function $E$, the $\lambda^{d}$ can be non unique (see Remark 2.5) and even the existence of a solution $v$ to (1.8) for some constant $\lambda^{d}$ is not known. 


\subsection{Main results}

For the applications that we have in mind, namely the study of the homogenization of dislocation dynamics, it is important to compute numerically a good approximation of the effective Hamiltonian $\lambda$ in equation (1.6). To this end, we consider approximate solutions of the discrete cell problem (1.8), and the goal of this paper is to provide some a posteriori error estimates between any discrete effective Hamiltonian and the continuous effective Hamiltonian $\lambda$. Concerning the effective Hamiltonian for local Hamilton-Jacobi equations, we refer the reader to [1],[8],[17],[22],[25] for error estimates and numerical computations; see also [2],[3] for a posteriori error estimates.

We start with the following definition.

Definition $1.2\left(\underline{\lambda}_{v}^{d}\right.$ and $\left.\bar{\lambda}_{v}^{d}\right)$

Let us consider a function $v: \mathbb{Z}^{N} \rightarrow \mathbb{R}$ satisfying condition (1.7). We call $\underline{\lambda}_{v}^{d}$ the maximal $\lambda^{d}$ such that

$$
\lambda^{d} \leq R[v]
$$

and we call $\bar{\lambda}_{v}^{d}$ the minimal $\lambda^{d}$ such that

$$
\lambda^{d} \geq R[v]
$$

where the inequalities are meant in the sense of viscosity for the scheme (see Definition 2.2).

Our first result is the following a posteriori Crandall-Lions type error estimate:

Theorem 1.3 (A posteriori $O(\sqrt{\Delta x})$ error estimate)

Under assumptions (1.3)-(1.4), let us consider a function $v: \mathbb{Z}^{N} \rightarrow \mathbb{R}$ satisfying condition (1.7). Then there exists a constant $C_{v}$, only depending on $v$, such that

$$
\underline{\lambda}_{v}^{d}-\lambda \leq C_{v} \sqrt{\Delta x} \quad \text { and } \quad \lambda-\bar{\lambda}_{v}^{d} \leq C_{v} \sqrt{\Delta x} .
$$

The constant $C_{v}$ can be computed explicitly from Theorem 4.6 and Remark 4.7.

We refer the reader to the pioneering work of Crandall and Lions [10] for Hamilton-Jacobi equations and also [4],[5],[12] for other similar estimates on different equations.

In dimension $N=1$, we can get a better error estimate under suitable assumptions. Let us first replace the index $I \in \mathbb{Z}^{N}$ by $i \in \mathbb{Z}$ to distinguish the special case of dimension one.

\section{Assumption (A)}

i) There exists some constants $l_{0}, L_{0}$ such that $0<l_{0} \leq \frac{v_{i+1}-v_{i}}{\Delta x} \leq L_{0}$ for all $i \in \mathbb{Z}$

ii) $\underline{\lambda}_{v}^{d} \geq 0$

iii) $\left(1+\frac{L_{0}}{l_{0}}\right) \Delta x \leq \frac{1}{2 L_{0}}$

iv) There exists a constant $C_{\mathcal{J}}$ such that the kernel $\mathcal{J}$ satisfies

$$
\mathcal{J}(z) \leq C_{\mathcal{J}} / z^{2} \quad \text { for all } z \in \mathbb{R} .
$$

Then we have the following result.

Theorem 1.4 (A posteriori $O(\Delta x)$ error estimate in 1D)

Under assumptions (1.3)-(1.4), let us consider a function $v: \mathbb{Z} \rightarrow \mathbb{R}$ satisfying condition (1.7) and assumption (A). Then we have the following error estimate

$$
\underline{\lambda}_{v}^{d}-\lambda \leq K_{v} \Delta x
$$

with

$$
K_{v}=L_{0}\left\{\operatorname{Lip}\left(c_{0}\right)+\frac{4}{3} \pi^{2} C_{\mathcal{J}} L_{0}^{2}\left(1+\frac{L_{0}}{l_{0}}\right)\right\}
$$


Another estimate can be obtained also for $\bar{\lambda}_{v}^{d}$ (see Theorem 5.1 for a precise statement).

The next natural question is: how to find a good candidate for $v$ in order to get a good approximation of $\lambda$ ? To answer to this question in any dimension $N \geq 1$, we recall that the effective Hamiltonian $\lambda$ for the continuous cell problem was constructed in [15] using the long time behaviour of the solution to the Cauchy problem (see (3.1)). Here a natural strategy is then to consider an implicit scheme for the discrete time evolution with the time step $\Delta t>0$ :

$$
\left\{\begin{array}{l}
\frac{v_{I}^{n+1}-v_{I}^{n}}{\Delta t}=R_{I}\left[v^{n+1}\right] \quad \text { and } v^{n+1} \text { satisfies }(1.7) \text { for } n \geq 0, \\
v_{I}^{0}=p \cdot x_{I} .
\end{array}\right.
$$

We have used an implicit scheme, instead of an explicit one, in order to be able to get an error estimate between any solution of the scheme and the solution of the corresponding partial differential equation. This fact was already remarked in [14]. The scheme (1.9) is not monotone, which means that a sub-solution may be above a super-solution at some points (see Definition 3.4 and Remark 3.5). This lack of comparison principle is due to the discontinuities in the definition of the scheme. In particular, there is no uniqueness of the solution to the scheme. Nevertheless we have the following result.

Theorem 1.5 (Minimal and maximal solutions for the time-evolution scheme) Under assumptions (1.3)-(1.4), the scheme (1.9) enjoys the following properties:

i) there exists a minimal solution $\underline{v}=\left(\underline{v}_{I}^{n}\right)$ and a maximal solution $\bar{v}=\left(\bar{v}_{I}^{n}\right)$ such that every solution $v=\left(v_{I}^{n}\right)$ satisfies

$$
\underline{v} \leq v \leq \bar{v}
$$

ii) there exists a solution $v$ such that the discrete time derivatives

$$
\underline{\mu}^{n}=\inf _{I \in \mathbb{Z}}\left(\frac{v_{I}^{n}-v_{I}^{n-1}}{\Delta t}\right) \quad \text { and } \quad \bar{\mu}^{n}=\sup _{I \in \mathbb{Z}}\left(\frac{v_{I}^{n}-v_{I}^{n-1}}{\Delta t}\right), \quad n \geq 1
$$

satisfy the following monotonicity property:

$$
\underline{\mu}^{n} \leq \underline{\mu}^{n+1} \leq \bar{\mu}^{n+1} \leq \bar{\mu}^{n}, \quad n \geq 1 .
$$

Remark 1.6 (Application to the improvement of the approximation of the effective Hamiltonian)

By definition we have

$$
\underline{\mu}^{n} \leq \underline{\lambda}_{v^{n}}^{d} \leq R\left[v^{n}\right] \quad \text { and } \quad \bar{\mu}^{n} \geq \bar{\lambda}_{v^{n}}^{d} \geq R\left[v^{n}\right] .
$$

From Theorem 1.5, we see that the gap $\bar{\mu}^{n}-\mu^{n}$ is a non-increasing function of $n$, and then the time evolution scheme provides a method to improve our numerical approximation of the effective Hamiltonian (using the error estimates given in Theorem 1.3 or Theorem 1.4).

\subsection{Organization of the paper}

The paper is organized as follows. In Section 2 we recall the notion of viscosity solutions for the cell problem, we introduce the corresponding numerical scheme and prove some properties it satisfies. Section 3 is devoted to the continuous evolution problem and its discrete version; we give in particular a sketch of the proof of Theorem 1.1. In Section 4 we prove an error estimate of Crandall-Lions type for the evolution problem and we deduce an a posteriori estimate for the cell problem (Theorem 1.3), while in Section 5 we improve the result in the one-dimensional case (Theorem 1.4). Section 6 concerns the numerical scheme for the evolution problem, we prove an existence result of discrete solutions and show how to contruct extremal solutions in practice (Theorem 1.5). Finally, in Section 7, we present some numerical simulations in one and two dimensions. 


\section{Viscosity solutions for the stationary problems}

\subsection{Viscosity solutions for the stationary PDE}

We adopt the notion of viscosity solution for non-local Hamilton-Jacobi equations introduced by Slepčev in [24] and then adapted to equation (1.6) in [15].

To this end we consider the upper and lower semicontinuous envelopes of the integer part $E$ in (1.2), respectively

$$
\begin{array}{ll}
E^{*}(\alpha)=k+\frac{1}{2} & \text { if } k \leq \alpha<k+1, \quad k \in \mathbb{Z}, \\
E_{*}(\alpha)=k+\frac{1}{2} & \text { if } k<\alpha \leq k+1, \quad k \in \mathbb{Z} .
\end{array}
$$

For a function $u: \mathbb{R}^{N} \rightarrow \mathbb{R}$ satisfying (1.1), we define the corresponding non-local velocities $c^{*}[u]$ and $c_{*}[u]$ as follows:

$$
c^{*}[u](x)=c_{0}(x)+\int_{\mathbb{R}^{N}} \mathcal{J}(z)\left\{E^{*}(u(x+z)-u(x))-p \cdot z\right\} d z
$$

and

$$
c_{*}[u](x)=c_{0}(x)+\int_{\mathbb{R}^{N}} \mathcal{J}(z)\left\{E_{*}(u(x+z)-u(x))-p \cdot z\right\} d z .
$$

We also denote by $u^{*}$ and $u_{*}$ the upper and lower semicontinuous envelopes of $u$, respectively. We give the definition of viscosity solution for the cell problem (1.6).

\section{Definition 2.1 (Viscosity solution for the stationary PDE)}

We consider a function $u: \mathbb{R}^{N} \rightarrow \mathbb{R}$ satisfying (1.1). We say that $u$ is a viscosity sub-solution of (1.6) if $u$ is upper semicontinuous and if for all $x_{0} \in \mathbb{R}^{N}$ and for all test functions $\varphi \in C^{1}\left(\mathbb{R}^{N}\right)$ such that $u-\varphi$ attains a local maximum at $x_{0}$, then we have

$$
\lambda \leq\left|\nabla \varphi\left(x_{0}\right)\right| c^{*}[u]\left(x_{0}\right) .
$$

Analogously we say that $u$ is a viscosity super-solution of (1.6) if $u$ is lower semicontinuous and if for all $x_{0} \in \mathbb{R}^{N}$ and for all test functions $\varphi \in C^{1}\left(\mathbb{R}^{N}\right)$ such that $u-\varphi$ attains a local minimum at $x_{0}$, then we have

$$
\lambda \geq\left|\nabla \varphi\left(x_{0}\right)\right| c_{*}[u]\left(x_{0}\right) .
$$

Then we say that $u$ is a viscosity solution of (1.6) if $u^{*}$ is a viscosity sub-solution and $u_{*}$ is a viscosity super-solution.

\subsection{Viscosity solutions for the stationary scheme}

In this subsection, we introduce the numerical scheme for which we define the notion of viscosity solution.

We consider a grid which is uniform in each dimension, i.e. we choose a discretization step in space $\Delta x$ with $\frac{1}{\Delta x} \in \mathbb{N}$ and nodes $x_{I}=\left(i_{1} \Delta x, \ldots, i_{N} \Delta x\right)$, where $I=\left(i_{1}, \ldots, i_{N}\right) \in \mathbb{Z}^{N}$. For every discrete function $v: \mathbb{Z}^{N} \rightarrow \mathbb{R}$ we denote by $v_{\sharp}$ its piecewise constant extension to $\mathbb{R}^{N}$, given by

$$
v_{\sharp}(x)=\sum_{I \in \mathbb{Z}^{N}} v_{I} \chi_{Q_{I}}(x),
$$

with

$$
Q_{I}=\left[x_{i_{1}}-\Delta x / 2, x_{i_{1}}+\Delta x / 2\right) \times \ldots \times\left[x_{i_{N}}-\Delta x / 2, x_{i_{N}}+\Delta x / 2\right) .
$$

By an abuse of notation, we also denote $v_{I}$ by $v\left(x_{I}\right)$.

\section{Discretization of the non-local velocity}

When $v$ satisfies (1.7), we discretize the non-local velocity $c[\cdot]=c_{0}+M_{p}[\cdot]$ as follows. For all $I \in \mathbb{Z}^{N}$, we define the discrete velocities

$$
\left(c^{*}\right)_{I}^{d}[v]:=c^{*}\left[v_{\sharp}\right]\left(x_{I}\right) \quad \text { and } \quad\left(c_{*}\right)_{I}^{d}[v]:=c_{*}\left[v_{\sharp}\right]\left(x_{I}\right) .
$$




\section{Discretization of the gradient}

On the other hand we approximate the gradients of discrete functions by considering the standard forward and backward first order differences:

$$
D_{k}^{+} v\left(x_{I}\right)=\frac{v\left(x_{I+e_{k}}\right)-v\left(x_{I}\right)}{\Delta x}, \quad D_{k}^{-} v\left(x_{I}\right)=\frac{v\left(x_{I}\right)-v\left(x_{I-e_{k}}\right)}{\Delta x}, \quad k=1, \ldots, N,
$$

where $I \pm e_{k}=\left(i_{1}, \ldots, i_{k-1}, i_{k} \pm 1, i_{k+1}, \ldots, i_{N}\right)$ and

$$
D v_{I}=\left(D^{+} v_{I}, D^{-} v_{I}\right) \quad \text { with } \quad D^{ \pm} v_{I}=\left(D_{1}^{ \pm} v\left(x_{I}\right), \ldots, D_{N}^{ \pm} v\left(x_{I}\right)\right) .
$$

For all $S=(P, Q) \in \mathbb{R}^{N} \times \mathbb{R}^{N}$, let us consider the following Osher, Sethian [21] discretization of the modulus of the gradient in an upwind fashion (see also [23] for a different discretization)

$$
\begin{aligned}
& G^{+}(S)=\left(\sum_{i=1}^{N}\left(\max \left\{P_{i}, 0\right\}\right)^{2}+\sum_{i=1}^{N}\left(\min \left\{Q_{i}, 0\right\}\right)^{2}\right)^{\frac{1}{2}}, \\
& G^{-}(S)=\left(\sum_{i=1}^{N}\left(\min \left\{P_{i}, 0\right\}\right)^{2}+\sum_{i=1}^{N}\left(\max \left\{Q_{i}, 0\right\}\right)^{2}\right)^{\frac{1}{2}}
\end{aligned}
$$

and for any velocity $c \in \mathbb{R}$, let us define the following continuous function:

$$
\mathcal{R}(c, S):= \begin{cases}c G^{+}(S) & \text { if } c \geq 0 \\ c G^{-}(S) & \text { if } c<0\end{cases}
$$

Then we introduce the following notion of viscosity solution.

\section{Definition 2.2 (Viscosity solution for the stationary scheme)}

Let $v: \mathbb{Z}^{N} \rightarrow \mathbb{R}$ be a function satisfting (1.7). We say that $v$ is a sub-solution of (1.8) if for all $I \in \mathbb{Z}^{N}$ we have

$$
\lambda^{d} \leq \mathcal{R}\left(\left(c^{*}\right)_{I}^{d}[v], D v_{I}\right)=: R_{I}^{*}[v] .
$$

We say that $v$ is a super-solution of (1.8) if for all $I \in \mathbb{Z}^{N}$ we have

$$
\lambda^{d} \geq \mathcal{R}\left(\left(c_{*}\right)_{I}^{d}[v], D v_{I}\right)=:\left(R_{*}\right)_{I}[v] .
$$

Then we say that $v$ is a solution of (1.8) if and only if it is a sub and a super solution.

Remark 2.3 The terminology of viscosity solutions may sound strange in the context of discrete schemes. See the proof of Theorem 4.3 step 4 for a justification.

\section{Remark 2.4 (Notation)}

When $v$ is a sub-solution of (1.8), we also write:

$$
\lambda^{d} \leq R[v],
$$

while when $v$ is a super-solution of (1.8), we also write:

$$
\lambda^{d} \geq R[v] .
$$

Those notations are used in Definition 1.2 and provide in particular a definition of a viscosity solution of equation (1.8).

\section{Remark 2.5 (Non uniqueness of $\lambda^{d}$ )}

In contrast with the continuous setting, the discrete effective Hamiltonian $\lambda_{d}$ is non unique in general, since the operators $R^{*}$ and $R_{*}$ are not the same. We now give such an example.

In dimension $N=1$ choose a kernel $\mathcal{J}$ such that $\mathcal{J}(z)>0$ for every $z \in \mathbb{R}$, take $c_{0}=0$ and $v_{i}=p i \Delta x$ with $0<p \Delta x \in \mathbb{Q}$. It follows that

$$
\left(c^{*}\right)_{i}^{d}[v] \equiv \text { const }=: C^{*}, \quad\left(c_{*}\right)_{i}^{d}[v] \equiv \text { const }=: C_{*} \quad \text { for all } i \in \mathbb{Z}
$$


and $C^{*}>C_{*}$. Moreover $\left|\frac{v_{i+1}-v_{i}}{\Delta x}\right|=p$. Then, by setting

$$
\underline{\lambda}^{d}=p C^{*} \quad \text { and } \quad \bar{\lambda}^{d}=p C_{*},
$$

we conclude that

$$
\left(R_{*}\right)_{i}[v]=\bar{\lambda}^{d}<\underline{\lambda}^{d}=R_{i}^{*}[v], \quad \text { for all } i \in \mathbb{Z},
$$

i.e., the pairs $\left(\bar{\lambda}^{d}, v\right)$ and $\left(\underline{\lambda}^{d}, v\right)$ are two different solutions of the cell problem (1.8).

Our scheme (1.9) is almost monotone, with the meaning that the operators $R^{*}$ and $R_{*}$ are monotone as it is stated in the following lemma.

\section{Lemma 2.6 (Monotonicity of $R^{*}$ and $R_{*}$ )}

Let $v, w$ be two discrete functions and suppose that

$$
v_{J} \leq w_{J} \quad \text { for all } J \in \mathbb{Z}^{N} .
$$

If there exists some index $I \in \mathbb{Z}^{N}$ such that $v_{I}=w_{I}$, then we have

$$
R_{I}^{*}[v] \leq R_{I}^{*}[w] \quad \text { and } \quad\left(R_{*}\right)_{I}[v] \leq\left(R_{*}\right)_{I}[w] .
$$

Remark 2.7 The scheme (1.9) is not truely monotone, because (2.7) and $v_{I}=w_{I}$ do not imply $R_{I}^{*}[v] \leq$ $\left(R_{*}\right)_{I}[w]$.

\section{Proof of Lemma 2.6.}

We restrict the proof to $R^{*}$, since it is the same for $R_{*}$. We first remark that $\left(c^{*}\right)_{I}^{d}[v] \leq\left(c^{*}\right)_{I}^{d}[w]$. In fact, by definition we get

$$
\left(c^{*}\right)_{I}^{d}[w]-\left(c^{*}\right)_{I}^{d}[v]=\sum_{J \in \mathbb{Z}^{N}}\left(\int_{Q_{J}} \mathcal{J}(z) d z\right)\left\{E^{*}\left(w_{I+J}-w_{I}\right)-E^{*}\left(v_{I+J}-v_{I}\right)\right\} .
$$

By assumption (2.7) we have

$$
w_{I+J}-w_{I}=w_{I+J}-v_{I} \geq v_{I+J}-v_{I} .
$$

Since the kernel $\mathcal{J}$ is non negative and the function $E^{*}$ is non decreasing, we conclude that the right hand side in (2.8) is non negative.

We now consider three cases, depending on the signs of $\left(c^{*}\right)_{I}^{d}[v]$ and $\left(c^{*}\right)_{I}^{d}[w]$ :

i) $\left(c^{*}\right)_{I}^{d}[v] \leq 0 \leq\left(c^{*}\right)_{I}^{d}[w]$. In this case the result follows trivially by the fact that the function $G^{ \pm}$is non negative.

ii) $0 \leq\left(c^{*}\right)_{I}^{d}[v] \leq\left(c^{*}\right)_{I}^{d}[w]$. In this case we have

$$
R_{I}^{*}[v]=\left(c^{*}\right)_{I}^{d}[v] G^{+}\left(D v_{I}\right) \quad \text { and } \quad R_{I}^{*}[w]=\left(c^{*}\right)_{I}^{d}[w] G^{+}\left(D w_{I}\right) .
$$

By assumption (2.7) we get

$$
D_{k}^{+} v_{I} \leq D_{k}^{+} w_{I} \quad \text { and } \quad D_{k}^{-} v_{I} \geq D_{k}^{-} w_{I} \quad \text { for } k=1, \ldots, N
$$

and then

$$
\max \left\{D_{k}^{+} v_{I}, 0\right\}^{2} \leq \max \left\{D_{k}^{+} w_{I}, 0\right\}^{2} \quad \text { and } \quad \min \left\{D_{k}^{-} v_{I}, 0\right\}^{2} \leq \min \left\{D_{k}^{-} w_{I}, 0\right\}^{2} .
$$

It follows that $G^{+}\left(D v_{I}\right) \leq G^{+}\left(D w_{I}\right)$ and this implies the result.

iii) $\left(c^{*}\right)_{I}^{d}[v] \leq\left(c^{*}\right)_{I}^{d}[w] \leq 0$. We have

$$
R_{I}^{*}[v]=\left(c^{*}\right)_{I}^{d}[v] G^{-}\left(D v_{I}\right) \quad \text { and } \quad R_{I}^{*}[w]=\left(c^{*}\right)_{I}^{d}[w] G^{-}\left(D w_{I}\right)
$$

and following the reasoning of step $(i i)$, we obtain $G^{-}\left(D v_{I}\right) \geq G^{-}\left(D w_{I}\right)$, which implies the result. The proof is complete. 


\section{Viscosity solutions for the time evolution problems}

In this section we recall the definition of viscosity solutions for the time evolution problems.

\subsection{Viscosity solutions for the time evolution PDE}

With the definition of the velocity $c[\cdot]$ given in (1.5), we now consider solutions $u$ of

$$
\begin{cases}u_{t}=|\nabla u| c[u(\cdot, t)] & \text { in } \mathbb{R}^{N} \times(0,+\infty), \\ u(\cdot, t) \text { satisfies }(1.1) \text { for all } t>0 & \\ u(x, 0)=u_{0}(x) & \text { for all } x \in \mathbb{R}^{N} .\end{cases}
$$

where the initial data $u_{0}$ satisfies $(1.1)$ and is globally Lipschitz-continuous on $\mathbb{R}^{N}$.

We recall that $c^{*}[\cdot]$ and $c_{*}[\cdot]$ are respectively defined in (2.1) and (2.2). Then we recall the following definition which was introduced in [15].

Definition 3.1 (Viscosity solutions for the time evolution PDE)

Let $u: \mathbb{R}^{N} \times \mathbb{R}^{+} \rightarrow \mathbb{R}$ be a function such that $u(t, \cdot)$ satisfies (1.1) for all $t>0$.

We say that $u$ is a viscosity sub-solution of (3.1) if $u$ is upper semicontinuous and satisfies

- $u(x, 0) \leq u_{0}(x)$ for all $x \in \mathbb{R}^{N}$;

- for all $\left(x_{0}, t_{0}\right) \in \mathbb{R}^{N} \times(0,+\infty)$ and for all test functions $\varphi \in C^{1}\left(\mathbb{R}^{N} \times(0,+\infty)\right)$ such that $u-\varphi$ attains a local maximum at $\left(x_{0}, t_{0}\right)$, then we have

$$
\varphi_{t}\left(x_{0}, t_{0}\right) \leq\left|\nabla_{x} \varphi\left(x_{0}, t_{0}\right)\right| c^{*}\left[u\left(\cdot, t_{0}\right)\right]\left(x_{0}\right) .
$$

Analogously we say that $u: \mathbb{R}^{N} \times \mathbb{R}^{+} \rightarrow \mathbb{R}$ is a viscosity super-solution of (3.1) if $u$ is lower semicontinuous and

- $u(x, 0) \geq u_{0}(x)$ for all $x \in \mathbb{R}^{N}$;

- for all $\left(x_{0}, t_{0}\right) \in \mathbb{R}^{N} \times(0,+\infty)$ and for all test functions $\varphi \in C^{1}\left(\mathbb{R}^{N} \times(0,+\infty)\right)$ such that $u-\varphi$ attains a local minimum at $\left(x_{0}, t_{0}\right)$, then we have

$$
\varphi_{t}\left(\left(x_{0}, t_{0}\right) \geq\left|\nabla_{x} \varphi\left(x_{0}, t_{0}\right)\right| c_{*}\left[u\left(\cdot, t_{0}\right)\right]\left(x_{0}\right) .\right.
$$

Then we say that $u$ is a viscosity solution of (3.1) if $u^{*}$ is a viscosity sub-solution and $u_{*}$ is a viscosity super-solution.

In the sequel we will need the following results (see [15] for details).

Theorem 3.2 (Comparison Principle) Let $u^{-}, u^{+}$be respectively a sub-solution and a super-solution of (3.1). Then $u^{-}(x, t) \leq u^{+}(x, t)$ for all $(x, t) \in \mathbb{R}^{N} \times(0,+\infty)$.

The main idea is that there is a comparison principle for the non-linear non-local right hand side of (3.1), essentially because the instability created by the discontinuity of the integer part $E$ is somehow compensated by the vanishing of the gradient at the same points (see the proofs of the comparison principle in [15],[7],[11]).

Theorem 3.3 (Existence-Uniqueness) For any $p \in \mathbb{R}^{N}$ there exists a unique continuous viscosity solution $u$ of the evolution problem (3.1). Moreover there exist two constants $\lambda \in \mathbb{R}$ and $C>0$ such that

$$
|u(x, t)-p \cdot x-\lambda t| \leq C, \quad \text { for } x \in \mathbb{R}^{N}, t \geq 0 .
$$

Now we give a sketch of the proof of Theorem 1.1, concerning the existence and uniqueness of the effective Hamiltonian.

Sketch of the proof of Theorem 1.1. 


\section{Step 1: Stability of viscosity solutions}

Let $u$ be the unique continuous viscosity solution of (3.1) given by Theorem 3.3, such that

$$
|u(x, t)-p \cdot x-\lambda t| \leq C, \quad \text { for } x \in \mathbb{R}^{N}, t \geq 0,
$$

for some $\lambda \in \mathbb{R}$ and $C>0$. We define for all $x \in \mathbb{R}^{N}$ and $t \in \mathbb{R}$

$$
\bar{u}(x, t)=\limsup _{\substack{n \rightarrow+\infty \\(y, s) \rightarrow(x, t)}}(u(y, s+n)-p \cdot y-\lambda n)+p \cdot x
$$

and

$$
\underline{u}(x, t)=\liminf _{\substack{n \rightarrow+\infty \\(y, s) \rightarrow(x, t)}}(u(y, s+n)-p \cdot y-\lambda n)+p \cdot x .
$$

By stability of viscosity solutions (see Proposition 4.2 in [15]) it follows that $\bar{u}, \underline{u}$ are respectively a subsolution and a super-solution of (3.1). Moreover we have

$$
|\bar{u}(x, t)-p \cdot x-\lambda t| \leq C \quad \text { and } \quad|\underline{u}(x, t)-p \cdot x-\lambda t| \leq C, \quad \text { for } x \in \mathbb{R}^{N}, t \in \mathbb{R} .
$$

\section{Step 2: Existence of a solution of the cell problem}

Define

$$
\overline{\bar{u}}(x)=\limsup _{\substack{y \rightarrow x \\ t \in \mathbb{R}}}(\bar{u}(y, t)-\lambda t) \quad \text { and } \quad \underline{\underline{u}}(x)=\liminf _{\substack{y \rightarrow x \\ t \in \mathbb{R}}}(\underline{u}(y, t)-\lambda t) .
$$

It is possible to prove that $\overline{\bar{u}}, \underline{\underline{u}}$ are respectively a sub-solution and a super-solution of the cell problem (1.6) corresponding to $\lambda$. Moreover

$$
|\overline{\bar{u}}(x)-p \cdot x| \leq C \quad \text { and } \quad|\underline{\underline{u}}(x)-p \cdot x| \leq C, \quad \text { for } x \in \mathbb{R}^{N},
$$

which implies

$$
\tilde{u}:=\overline{\bar{u}}-2 C \leq \underline{\underline{u}} .
$$

Since the cell problem is invariant with respect to the addition of constants, it follows that $\tilde{u}$ is still a sub-solution. Then, by applying the Perron's method, we conclude that there exists a solution $U$ of (1.6), satisfying (1.1) and such that

$$
\tilde{u} \leq U \leq \underline{\underline{u}} \quad \text { and } \quad|U(x)-p \cdot x| \leq 3 C .
$$

Step 3: Uniqueness of $\lambda$

By contradiction assume that $\left(\lambda_{1}, U_{1}\right)$ and $\left(\lambda_{2}, U_{2}\right)$ are two solutions of the cell problem (1.6), with $\lambda_{1}<\lambda_{2}$ and

$$
\left|U_{1}(x)-p \cdot x\right| \leq C_{0}, \quad\left|U_{2}(x)-p \cdot x\right| \leq C_{0}, \quad \text { for some constant } C_{0}>0 .
$$

Now define

$$
u_{1}(x, t)=U_{1}(x)+\lambda_{1} t+2 C_{0} \quad \text { and } \quad u_{2}(x, t)=U_{2}(x)+\lambda_{2} t .
$$

It follows that $u_{1}$ and $u_{2}$ are two viscosity solutions of the equation (3.1) with $u_{1}(\cdot, 0) \geq u_{2}(\cdot, 0)$. Then, by comparision principle (Theorem 3.2), we get $u_{1}(x, t) \geq u_{2}(x, t)$ for all $x \in \mathbb{R}^{N}$ and $t \geq 0$, i.e.,

$$
U_{1}(x)+\lambda_{1} t+2 C_{0} \geq U_{2}(x)+\lambda_{2} t .
$$

Dividing by $t$ and taking the limit as $t$ goes to infinity, we conclude that $\lambda_{1} \geq \lambda_{2}$, which is absurd. The proof is complete.

\subsection{Viscosity solutions for the time evolution scheme}

We consider solutions of the time evolution scheme

$$
\left\{\begin{array}{l}
\frac{v_{I}^{n+1}-v_{I}^{n}}{\Delta t}=R_{I}\left[v^{n+1}\right], \quad I \in \mathbb{Z}^{N}, n \in \mathbb{N} \\
v^{n+1} \text { satisfies (1.7) for } n \in \mathbb{N}, \\
v_{I}^{0}=\left(v_{0}\right)_{I}, \quad I \in \mathbb{Z}^{N},
\end{array}\right.
$$

where the initial data $v_{0}$ is assumed to satisfy (1.7). We recall that the operators $R^{*}$ and $R_{*}$ are defined in Definition 2.2. 
Definition 3.4 (Viscosity solutions for the time evolution scheme)

Let us consider a function $v: \mathbb{Z}^{N} \times \mathbb{N} \rightarrow \mathbb{R}$ such that $v^{n}$ satisfies (1.7) for every $n \in \mathbb{N}$. We say that $v$ is a sub-solution of (3.4) if for all $I \in \mathbb{Z}^{N}$ and $n \in \mathbb{N}$ we have

$$
\frac{v_{I}^{n+1}-v_{I}^{n}}{\Delta t} \leq R_{I}^{*}\left[v^{n+1}\right]
$$

We say that $v$ is a super-solution of (3.4) if for all $I \in \mathbb{Z}^{N}$ and $n \in \mathbb{N}$ we have

$$
\frac{v_{I}^{n+1}-v_{I}^{n}}{\Delta t} \geq\left(R_{*}\right)_{I}\left[v^{n+1}\right]
$$

Then we say that $v$ is a solution of (3.4) if and only if it is a sub and a super solution.

\section{Remark 3.5 (No comparison principle for the time evolution scheme)}

With the same assumptions of Remark 2.5 just define

$$
\underline{v}_{i}^{n}=v_{i}+\underline{\lambda}^{d} n \Delta t \quad \text { and } \quad \bar{v}_{i}^{n}=v_{i}+\bar{\lambda}^{d} n \Delta t .
$$

It follows that $\underline{v}, \bar{v}$ are respectively a sub-solution and a super-solution of the scheme (3.4) with initial datum $v_{0}=v$. In particular they coincide for $n=0$, but $\underline{v}^{n}>\bar{v}^{n}$ for all $n \geq 1$, i.e., in contrast with the continuous setting, the comparison principle does not hold for the scheme. This is due to the fact that the instability of the discontinuity of the integer part $E$ can not be here compensated by the vanishing of the gradient, because the discrete gradient can be non zero at the same points.

\section{Crandall-Lions type error estimates}

This section is devoted to one of the main results of this paper: we first prove an error estimate of CrandallLions type between the viscosity solution for the continuous evolution problem (3.1) and the discrete solutions of the scheme (3.4). Then we apply such estimate to obtain an analogous error estimate between the effective Hamiltonian of the cell problem (1.6) and the discrete solutions of the corresponding scheme (1.8).

\subsection{Estimate for the time evolution problem}

We start with some preliminary results, in particular we prove the existence of barriers for both the continuous and the discrete evolution problem.

\section{Proposition 4.1 (Continuous barriers)}

Let $u_{0}(x)=\tilde{u}_{0}(x)+p \cdot x$, where $\tilde{u}_{0}$ is a Lipschitz continuous and $\mathbb{Z}^{N}$-periodic function. Let $C_{u_{0}}$ be a constant such that

$$
\left.C_{u_{0}} \geq \max \left\{\left\|c_{*}\left[u_{0}\right]\right\|_{\infty},\left\|c^{*}\left[u_{0}\right]\right\|_{\infty}\right\}\right)\left\|\nabla u_{0}\right\|_{\infty}
$$

and define

$$
u^{ \pm}(x, t)=u_{0}(x) \pm C_{u_{0}} t, \quad \text { for all }(x, t) \in \mathbb{R}^{N} \times(0,+\infty) .
$$

Then the unique viscosity solution $u$ of (3.1) with initial datum $u_{0}$ satisfies

$$
u^{-}(x, t) \leq u(x, t) \leq u^{+}(x, t), \quad \text { for all }(x, t) \in \mathbb{R}^{N} \times(0,+\infty) .
$$

Proof.

It is easy to see that $u^{-}, u^{+}$are respectively a sub-solution and a super-solution of (3.1). Then we conclude by applying the comparison principle (Theorem 3.2) to $u, u^{+}$and $u^{-}, u$.

\section{Proposition 4.2 (Discrete barriers)}

Let $v_{0}$ satisfying (1.7). Let $C_{v_{0}}$ be a constant such that

$$
C_{v_{0}} \geq \max \left\{\left\|R_{*}\left[v_{0}\right]\right\|_{\infty},\left\|R^{*}\left[v_{0}\right]\right\|_{\infty}\right\}
$$


and define

$$
\left(v^{ \pm}\right)_{I}^{n}=\left(v_{0}\right)_{I} \pm C_{v_{0}} n \Delta t, \quad \text { for all } I \in \mathbb{Z}^{N}, n \in \mathbb{N} .
$$

Then every solution $v$ of the scheme (3.4), with initial datum $v_{0}$ satisfies

$$
\left(v^{-}\right)_{I}^{n} \leq v_{I}^{n} \leq\left(v^{+}\right)_{I}^{n} \quad \text { for all } I \in \mathbb{Z}^{N}, n \in \mathbb{N} .
$$

Proof.

We restrict the proof to the case of sub-solutions, since it is similar for super-solutions. Let $v$ be a sub-solution of the scheme, i.e.,

$$
\left\{\begin{array}{l}
\frac{v_{I}^{n}-v_{I}^{n-1}}{\Delta t} \leq R_{I}^{*}\left[v^{n}\right], \quad I \in \mathbb{Z}^{N}, n \in \mathbb{N} \backslash\{0\} \\
v_{I}^{0}=\left(v_{0}\right)_{I}, \quad I \in \mathbb{Z}^{N}
\end{array}\right.
$$

We want to prove by induction that $v_{I}^{n} \leq\left(v^{+}\right)_{I}^{n}$ for every $n \in \mathbb{N}$. The case $n=0$ is trivial, since $v_{I}^{0}=$ $\left(v_{0}\right)_{I}=\left(v^{+}\right)_{I}^{0}$ by definition. Now let $n \in \mathbb{N}$ and suppose that $v_{I}^{k} \leq\left(v^{+}\right)_{I}^{k}$ for all $k=0, \ldots, n-1$.

We set

$$
M=\sup _{I \in \mathbb{Z}^{N}}\left(v_{I}^{n}-\left(v^{+}\right)_{I}^{n}\right)=v_{I_{0}}^{n}-\left(v^{+}\right)_{I_{0}}^{n},
$$

where the supremum $M$ is achieved at a point $I_{0} \in \mathbb{Z}^{N}$ since both $v$ and $v^{+}$are of the form periodic plus linear with the same slope $p$. Then we have $v_{I}^{n}-M \leq\left(v^{+}\right)_{I}^{n}$ for all $I \in \mathbb{Z}^{N}$ and $v_{I_{0}}^{n}-M=\left(v^{+}\right)_{I_{0}}^{n}$. By Lemma 2.6 it follows that

$$
R_{I_{0}}^{*}\left[v^{n}-M\right] \leq R_{I_{0}}^{*}\left[\left(v^{+}\right)^{n}\right] .
$$

Then by the invariance of $R^{*}$ with respect to the addition of constants, we obtain

$$
\frac{v_{I_{0}}^{n}-v_{I_{0}}^{n-1}}{\Delta t} \leq R_{I_{0}}^{*}\left[v^{n}\right]=R_{I_{0}}^{*}\left[v^{n}-M\right] \leq R_{I_{0}}^{*}\left[\left(v^{+}\right)^{n}\right]=R_{I_{0}}^{*}\left[v_{0}\right] \leq C_{v_{0}}
$$

and this, together with the inductive assumption, implies

$$
v_{I_{0}}^{n} \leq v_{I_{0}}^{n-1}+\Delta t C_{v_{0}} \leq\left(v^{+}\right)_{I_{0}}^{n-1}+\Delta t C_{v_{0}}=\left(v^{+}\right)_{I_{0}}^{n} .
$$

We conclude that

$$
M=\sup _{I \in \mathbb{Z}^{N}}\left(v_{I}^{n}-\left(v^{+}\right)_{I}^{n}\right) \leq 0
$$

and then

$$
v_{I}^{n} \leq\left(v^{+}\right)_{I}^{n} \quad \text { for all } I \in \mathbb{Z}^{N} .
$$

The proof is complete.

Given a discrete function $v: \mathbb{Z}^{N} \times \mathbb{N} \rightarrow \mathbb{R}$ we denote by $v_{\sharp}$ its piecewise constant extension in space and time to $\mathbb{R}^{N} \times(0,+\infty)$, i.e., with a little abuse of notation in comparison to (2.5),

$$
v_{\sharp}(x, t)=\sum_{I \in \mathbb{Z}^{N}, n \in \mathbb{N}} v_{I}^{n} \chi_{Q_{I} \times\left[t_{n}, t_{n+1}\right)}(x, t),
$$

with $t_{n}=n \Delta t$. We also adopt the following notation: for $T>0$,

$$
\mathcal{G}^{\Delta x}\left(v_{\sharp}\right)=\sup _{\substack{|x-y| \geq \Delta x \\ t \in\left\{0, \ldots, t_{n_{T}}\right\}}} \frac{\left|v_{\sharp}(x, t)-v_{\sharp}(y, t)\right|}{|x-y|},
$$

where $n_{T}$ is the highest integer such that $n_{T} \Delta t \leq T$, and

$$
\mathcal{G}^{\Delta t}\left(v_{\sharp}\right)=\sup _{\substack{|t-s| \geq \Delta t \\ t, s \in\left\{0, \ldots, t_{n_{T}}\right\} \\ x \in \mathbb{R}^{N}}} \frac{\left|v_{\sharp}(x, t)-v_{\sharp}(x, s)\right|}{|t-s|} .
$$


Finally we introduce the following auxiliary velocities

$$
\begin{aligned}
& \tilde{c}_{*}\left[v_{\sharp}, x_{I}\right](x, t)=c_{0}(x)+\int_{\mathbb{R}^{N}} \mathcal{J}(y-x)\left\{E_{*}\left(v_{\sharp}(y, t)-v_{\sharp}\left(x_{I}, t\right)\right)-p \cdot(y-x)\right\} d y, \\
& \tilde{c}^{*}\left[v_{\sharp}, x_{I}\right](x, t)=c_{0}(x)+\int_{\mathbb{R}^{N}} \mathcal{J}(y-x)\left\{E^{*}\left(v_{\sharp}(y, t)-v_{\sharp}\left(x_{I}, t\right)\right)-p \cdot(y-x)\right\} d y
\end{aligned}
$$

and define

$$
\begin{aligned}
& \mathcal{L}\left(\tilde{c}_{*}\left[v_{\sharp}\right]\right)=\sup _{I \in \mathbb{Z}, t \in[0, T]} \operatorname{Lip}\left(\tilde{c}_{*}\left[v_{\sharp}, x_{I}\right](\cdot, t)\right), \\
& \mathcal{L}\left(\tilde{c}^{*}\left[v_{\sharp}\right]\right)=\sup _{I \in \mathbb{Z}, t \in[0, T]} \operatorname{Lip}\left(\tilde{c}^{*}\left[v_{\sharp}, x_{I}\right](\cdot, t)\right) .
\end{aligned}
$$

Now we can state the error estimate.

\section{Theorem 4.3 (Crandall-Lions type error estimate)}

Let $N \geq 1, T \geq 1$. Let $u$ be a continuous sub-solution of (3.1) with Lipschitz initial datum $u_{0}$ and $v$ a discrete super-solution of the scheme (3.4) with initial datum $v_{0}$. There exists a positive constant $K_{v_{\sharp}, u_{0}, v_{0}}$, which depends on $u_{0}, v_{0}, v_{\sharp}, N, p,\left\|c_{0}\right\|_{W^{1, \infty}\left(\mathbb{R}^{N}\right)},\|\mathcal{J}\|_{W^{1,1}\left(\mathbb{R}^{N}\right)}$, such that the following error estimate holds:

$$
\sup _{\mathbb{R}^{N} \times\left\{0, \ldots, t_{n_{T}}\right\}}\left(u-v_{\sharp}\right) \leq \sup _{\mathbb{R}^{N}}\left(u_{0}-\left(v_{0}\right)_{\sharp}\right)+K_{v_{\sharp}, u_{0}, v_{0}}(\Delta x+\Delta t+\sqrt{\Delta x+\Delta t})+\eta^{*} T,
$$

where $t_{n}=n \Delta t, n_{T}$ is the highest integer such that $n_{T} \Delta t \leq T$ and

$$
\eta^{*}=2 \sqrt{2 N} \mathcal{G}^{\Delta x}\left(v_{\sharp}\right)\left\|c_{*}\left[v_{\sharp}\right]\right\|_{\infty} \mathbf{X}(1+\mathbf{X}(1+\sqrt{N} / 2))^{2},
$$

with

$$
\mathbf{X}=\sqrt{\frac{\mathcal{L}\left(\tilde{c}_{*}\left[v_{\sharp}\right]\right) \varepsilon}{\left\|c_{*}\left[v_{\sharp}\right]\right\|_{\infty} \sqrt{N / 2}}}, \quad \varepsilon=\Delta x+\frac{\Delta t}{\left\|c_{*}\left[v_{\sharp}\right]\right\|_{\infty} \sqrt{2 N}} .
$$

To do the proof of Theorem 4.3, we will need the following result.

\section{Lemma 4.4 (Viscosity inequality at time $T$ )}

Let $u$ be a continuous sub-solution of equation (3.1) and take $T>0$. For every test function $\varphi \in C^{1}\left(\mathbb{R}^{N} \times\right.$ $(0,+\infty))$ such that

$$
\max _{\mathbb{R}^{N} \times[0, T]} u-\varphi=u\left(x_{0}, T\right)-\varphi\left(x_{0}, T\right) \quad \text { for some } x_{0} \in \mathbb{R}^{N},
$$

the following viscosity inequality holds:

$$
\varphi_{t}\left(x_{0}, T\right) \leq\left|\nabla_{x} \varphi\left(x_{0}, T\right)\right| c^{*}[u(\cdot, T)]\left(x_{0}\right) .
$$

Sketch of the proof.

The idea is to introduce the following perturbation of the test function $\varphi$,

$$
\varphi^{\eta}(x, t)=\varphi(x, t)+\frac{\eta}{T-t}, \quad \eta>0,
$$

which forces the difference $u-\varphi^{\eta}$ to attain a local maximum at a point $\left(x_{\eta}, t_{\eta}\right)$ such that $t_{\eta}<T$ and $\left(x_{\eta}, t_{\eta}\right) \stackrel{\eta \rightarrow 0}{\longrightarrow}\left(x_{0}, T\right)$. It follows that $\varphi^{\eta}$ is a good test function for the viscosity inequality at $\left(x_{\eta}, t_{\eta}\right)$, i.e.,

$$
\varphi_{t}^{\eta}\left(x_{\eta}, t_{\eta}\right) \leq\left|\nabla_{x} \varphi^{\eta}\left(x_{\eta}, t_{\eta}\right)\right| c^{*}\left[u\left(\cdot, t_{\eta}\right)\right]\left(x_{\eta}\right) .
$$

We conclude by taking $\eta \rightarrow 0$ and using the fact that $(x, t) \mapsto c^{*}[u(\cdot, t)](x)$ is an upper-semicontinuous function.

Proof of Theorem 4.3.

We follow the method introduced by Crandall and Lions in [10] and then adapted in [5],[12]. One of the 
differences is that we get here a posteriori estimates instead of a priori estimates.

We first assume that

$$
u_{0}\left(x_{I}\right) \geq\left(v_{0}\right)_{\sharp}\left(x_{I}\right) \quad \text { for all } I \in \mathbb{Z}^{N}
$$

and define

$$
\mu_{0}=\sup _{\mathbb{R}^{N}}\left(u_{0}-\left(v_{0}\right)_{\sharp}\right) \geq 0 .
$$

We duplicate the variables by defining, for every $\gamma>0$ and $\eta \geq 0$,

$$
\Psi \equiv \Psi_{\eta}^{\gamma}(x, t, y, s)=u(x, t)-v_{\sharp}(y, s)-\frac{|x-y|^{2}}{2 \gamma}-\frac{|t-s|^{2}}{2 \gamma}-\eta t
$$

and we set

$$
M=\sup \left\{\Psi_{\eta}^{\gamma}(x, t, y, s) \quad: \quad x, y \in \mathbb{R}^{N}, t \in[0, T], s \in\left\{0, \ldots, t_{n_{T}}\right\}\right\} .
$$

We remark that the supremum $M$ is achieved at a point $(\bar{x}, \bar{t}, \bar{y}, \bar{s}) \in \mathbb{R}^{N} \times[0, T] \times \mathbb{R}^{N} \times\left\{0, \ldots, t_{n_{T}}\right\}$ since both $x \mapsto u(x, t)-p \cdot x$ and $x \mapsto v_{\sharp}(x, t)-p \cdot x$ are $\mathbb{Z}^{N}$-periodic by assumption.

The proof is splitted in several steps.

Step 1: estimates on $u$ and $v_{\sharp}$

By Proposition 4.1 the sub-solution $u$ satisfies

$$
u(x, t)-u_{0}(x) \leq C_{u_{0}} t \quad \text { for all } x \in \mathbb{R}^{N} \text { and } t \in[0, T],
$$

where the constant $C_{u_{0}}$ is given by (4.1).

Similarly by Proposition 4.2 the super-solution $v$ satisfies

$$
\left(v_{0}\right)_{\sharp}(y)-v_{\sharp}(y, s) \leq C_{v_{0}} s \quad \text { for all } y \in \mathbb{R}^{N} \text { and } s \in\left\{0, \ldots, t_{n_{T}}\right\} .
$$

where the constant $C_{v_{0}}$ is given by (4.2).

\section{Step 2: estimate of the maximum point of $\Psi$}

We first look for an estimate of $|\bar{x}-\bar{y}|$. From the inequality $\Psi(\bar{x}, \bar{t}, \bar{x}, \bar{s}) \leq \Psi(\bar{x}, \bar{t}, \bar{y}, \bar{s})$ we get

$$
\frac{|\bar{x}-\bar{y}|^{2}}{2 \gamma} \leq v_{\sharp}(\bar{x}, \bar{s})-v_{\sharp}(\bar{y}, \bar{s}) .
$$

If $|\bar{x}-\bar{y}| \geq \Delta x$ we obtain

$$
\frac{|\bar{x}-\bar{y}|^{2}}{2 \gamma} \leq \mathcal{G}^{\Delta x}\left(v_{\sharp}\right)|\bar{x}-\bar{y}|,
$$

with $\mathcal{G}^{\Delta x}\left(v_{\sharp}\right)$ given by $(4.3)$. It follows that

$$
|\bar{x}-\bar{y}| \leq 2 \mathcal{G}^{\Delta x}\left(v_{\sharp}\right) \gamma+\Delta x .
$$

Similarly we obtain an estimate of $|\bar{t}-\bar{s}|$. In fact, let $\tilde{t}$ be the projection of $\bar{t}$ on the time grid, namely the point $\tilde{t}=\tilde{n} \Delta t, \tilde{n} \in \mathbb{N}$, such that $|\tilde{t}-\bar{t}| \leq \Delta t$. From the inequality $\Psi(\bar{x}, \bar{t}, \bar{y}, \tilde{t}) \leq \Psi(\bar{x}, \bar{t}, \bar{y}, \bar{s})$, we get

$$
\frac{|\bar{t}-\bar{s}|^{2}}{2 \gamma} \leq \frac{|\tilde{t}-\bar{t}|^{2}}{2 \gamma}+v_{\sharp}(\bar{y}, \tilde{t})-v_{\sharp}(\bar{y}, \bar{s}) \leq \frac{|\tilde{t}-\bar{t}|^{2}}{2 \gamma}+\mathcal{G}^{\Delta t}\left(v_{\sharp}\right)|\tilde{t}-\bar{s}| \leq \frac{(\Delta t)^{2}}{2 \gamma}+\mathcal{G}^{\Delta t}\left(v_{\sharp}\right)(|\bar{t}-\bar{s}|+\Delta t),
$$

with $\mathcal{G}^{\Delta t}\left(v_{\sharp}\right)$ given by (4.4). If $|\bar{t}-\bar{s}| \geq 2 \Delta t$ we obtain

$$
\frac{|\bar{t}-\bar{s}|^{2}}{2 \gamma} \leq \frac{1}{4} \frac{|\bar{t}-\bar{s}|^{2}}{2 \gamma}+\frac{3}{2} \mathcal{G}^{\Delta t}\left(v_{\sharp}\right)|\bar{t}-\bar{s}|,
$$

i.e.,

$$
\frac{|\bar{t}-\bar{s}|^{2}}{2 \gamma} \leq 2 \mathcal{G}^{\Delta t}\left(v_{\sharp}\right)|\bar{t}-\bar{s}|,
$$


and then we conclude

$$
|\bar{t}-\bar{s}| \leq 4 \mathcal{G}^{\Delta t}\left(v_{\sharp}\right) \gamma+2 \Delta t .
$$

Now we claim that if $\eta$ is big enough then $\bar{t}=0$ or $\bar{s}=0$. We proceed by contradiction and assume that $\bar{t}>0$ and $\bar{s}>0$.

\section{Step 3: the continuous viscosity inequality}

We set

$$
\varphi(x, t)=v_{\sharp}(\bar{y}, \bar{s})+\frac{|x-\bar{y}|^{2}}{2 \gamma}+\frac{|t-\bar{s}|^{2}}{2 \gamma}+\eta t .
$$

Then $u-\varphi$ achieves a maximum at the point $(\bar{x}, \bar{t})$ and $u$ is a sub-solution of equation (3.1). It follows that

$$
\varphi_{t}(\bar{x}, \bar{t}) \leq c^{*}[u](\bar{x}, \bar{t})|\nabla \varphi(\bar{x}, \bar{t})|,
$$

where we denote $c^{*}[u(\cdot, \bar{t})](\bar{x})$ by $c^{*}[u](\bar{x}, \bar{t})$ in order to simplify the presentation. Then we obtain

$$
\eta+\frac{\bar{t}-\bar{s}}{\gamma} \leq c^{*}[u](\bar{x}, \bar{t}) \frac{|\bar{x}-\bar{y}|}{\gamma} .
$$

We remark here a crucial point: if the maximum of $u-\varphi$ is achieved at the final time $\bar{t}=T$, in general it is not a local maximum, since it is not excluded that $u$ is above $\varphi$ for $t>T$. Nevertheless the viscosity inequality (4.14) still holds by Lemma 4.4.

\section{Step 4: the discrete viscosity inequality}

Let $\bar{y}_{I}$ be the grid point such that $\bar{y} \in Q_{I}$ and $\bar{n} \in \mathbb{N}$ such that $\bar{s}=\bar{n} \Delta t$. For every $y \in \mathbb{R}^{N}$ and $s \geq \bar{s}-\Delta t$ we set

$$
\varphi(y, s)=-\frac{|\bar{x}-y|^{2}}{2 \gamma}-\frac{|\bar{t}-s|^{2}}{2 \gamma} .
$$

From the inequality $\Psi(\bar{x}, \bar{t}, y, s) \leq \Psi(\bar{x}, \bar{t}, \bar{y}, \bar{s})$ we get

$$
v_{\sharp}(\bar{y}, \bar{s})-v_{\sharp}(y, s) \leq \varphi(\bar{y}, \bar{s})-\varphi(y, s) .
$$

Since $v_{\sharp}$ is the piecewise constant extension of a super-solution $v$ of the scheme (3.4), for $y=\bar{y}$ and $s=\bar{s}-\Delta t$ we have

$$
\begin{gathered}
\frac{\varphi(\bar{y}, \bar{s})-\varphi(\bar{y}, \bar{s}-\Delta t)}{\Delta t} \geq \frac{v_{\sharp}\left(\bar{y}_{I}, \bar{n} \Delta t\right)-v_{\sharp}\left(\bar{y}_{I},(\bar{n}-1) \Delta t\right)}{\Delta t}= \\
=\frac{v_{I}^{\bar{n}}-v_{I}^{\bar{n}-1}}{\Delta t} \geq\left(R_{*}\right)_{I}\left[v^{\bar{n}}\right] .
\end{gathered}
$$

Moreover for $y_{ \pm}=\bar{y} \pm e_{k} \Delta x, s=\bar{s}$ and $k=1, \ldots, N$ it follows that

$$
\begin{aligned}
& D_{k}^{+} v_{I}^{\bar{n}} \geq D_{k}^{+} \varphi(\bar{y}, \bar{s}):=\frac{\varphi\left(y_{+}, \bar{s}\right)-\varphi(\bar{y}, \bar{s})}{\Delta x} \quad \Longrightarrow \quad G^{+}\left(D v_{I}^{\bar{n}}\right) \geq G^{+}(D \varphi(\bar{y}, \bar{s})) \\
& D_{k}^{-} v_{I}^{\bar{n}} \leq D_{k}^{-} \varphi(\bar{y}, \bar{s}):=\frac{\varphi(\bar{y}, \bar{s})-\varphi\left(y_{-}, \bar{s}\right)}{\Delta x} \quad \Longrightarrow \quad G^{-}\left(D v_{I}^{\bar{n}}\right) \leq G^{-}(D \varphi(\bar{y}, \bar{s}))
\end{aligned}
$$

where

$$
D \varphi(\bar{y}, \bar{s})=\left(D^{+} \varphi(\bar{y}, \bar{s}), D^{-} \varphi(\bar{y}, \bar{s})\right) \quad \text { with } \quad D^{ \pm} \varphi(\bar{y}, \bar{s})=\left(D_{1}^{ \pm} \varphi(\bar{y}, \bar{s}), \ldots, D_{N}^{ \pm} \varphi(\bar{y}, \bar{s})\right)
$$

Then

$$
\frac{\varphi(\bar{y}, \bar{s})-\varphi(\bar{y}, \bar{s}-\Delta t)}{\Delta t} \geq c_{*}\left[v_{\sharp}\right]\left(\bar{y}_{I}, \bar{s}\right) G^{s\left[v_{\sharp}\right]}(D \varphi(\bar{y}, \bar{s})) .
$$

where $s\left[v_{\sharp}\right]=\operatorname{sign}\left(c_{*}\left[v_{\sharp}\right]\left(\bar{y}_{I}, \bar{s}\right)\right)$. Finally we set

$$
\bar{q}_{x}=\frac{\bar{x}-\bar{y}}{\gamma}, \quad \bar{q}_{t}=\frac{\bar{t}-\bar{s}}{\gamma}
$$


and by straightforward computations on discrete derivatives of $\varphi$ we obtain

$$
\bar{q}_{t}+\frac{\Delta t}{2 \gamma} \geq c_{*}\left[v_{\sharp}\right]\left(\bar{y}_{I}, \bar{s}\right) G^{s\left[v_{\sharp}\right]}\left(\bar{q}_{x}-\frac{\overrightarrow{\Delta x}}{2 \gamma}, \bar{q}_{x}+\frac{\overrightarrow{\Delta x}}{2 \gamma}\right),
$$

with $\overrightarrow{\Delta x}=\Delta x(1, \ldots, 1)$

\section{Step 5: subtracting the viscosity inequalities}

From (4.14) and (4.15) we get

$$
\eta \leq \frac{\Delta t}{2 \gamma}+\left(c^{*}[u](\bar{x}, \bar{t})-c_{*}\left[v_{\sharp}\right]\left(\bar{y}_{I}, \bar{s}\right)\right)\left|\bar{q}_{x}\right|+\left|c_{*}\left[v_{\sharp}\right]\left(\bar{y}_{I}, \bar{s}\right)\right|\left|G^{s\left[v_{\sharp}\right]}\left(\bar{q}_{x}-\frac{\overrightarrow{\Delta x}}{2 \gamma}, \bar{q}_{x}+\frac{\overrightarrow{\Delta x}}{2 \gamma}\right)-\right| \bar{q}_{x}|| .
$$

The latter term can be estimated as follows. Since $\left|\bar{q}_{x}\right|=G^{ \pm}\left(\bar{q}_{x}, \bar{q}_{x}\right)$ and the discrete gradients $G^{ \pm}$are 1-Lipschitz continuous functions we have

$$
\left|c_{*}\left[v_{\sharp}\right]\left(\bar{y}_{I}, \bar{s}\right)\right|\left|G^{s\left[v_{\sharp}\right]}\left(\bar{q}_{x}-\frac{\overrightarrow{\Delta x}}{2 \gamma}, \bar{q}_{x}+\frac{\overrightarrow{\Delta x}}{2 \gamma}\right)-\right| \bar{q}_{x}|| \leq \sqrt{2}\left\|c_{*}\left[v_{\sharp}\right]\right\|_{\infty}\left|\frac{\overrightarrow{\Delta x}}{2 \gamma}\right|=\sqrt{\frac{N}{2}}\left\|c_{*}\left[v_{\sharp}\right]\right\|_{\infty} \frac{\Delta x}{\gamma} .
$$

Step 6: estimate on the difference of the velocities when $|\bar{x}-\bar{y}| \neq 0$

From the inequality $\Psi(y, \bar{t}, y, \bar{s}) \leq \Psi(\bar{x}, \bar{t}, \bar{y}, \bar{s})$ we get for every $y \in \mathbb{R}^{N}$

$$
\underbrace{u(y, \bar{t})-u(\bar{x}, \bar{t})}_{\alpha} \leq \underbrace{v_{\sharp}(y, \bar{s})-v_{\sharp}(\bar{y}, \bar{s})}_{\beta}-\underbrace{\frac{|\bar{x}-\bar{y}|^{2}}{2 \gamma}}_{\varepsilon} .
$$

By monotonicity of $E^{*}$ we have $E^{*}(\alpha) \leq E^{*}(\beta-\varepsilon)$. Moreover $E^{*}(\beta-\varepsilon) \leq E_{*}(\beta)$, since $\varepsilon>0$. Then, by changing variables, we obtain

$$
\begin{gathered}
c^{*}[u](\bar{x}, \bar{t})=c_{0}(\bar{x})+\int_{\mathbb{R}^{N}} \mathcal{J}(y-\bar{x})\left\{E^{*}(u(y, \bar{t})-u(\bar{x}, \bar{t}))-p \cdot(y-\bar{x})\right\} d y \leq \\
\leq c_{0}(\bar{x})+\int_{\mathbb{R}^{N}} \mathcal{J}(y-\bar{x})\left\{E_{*}\left(v_{\sharp}(y, \bar{s})-v_{\sharp}\left(\bar{y}_{I}, \bar{s}\right)\right)-p \cdot(y-\bar{x})\right\} d y
\end{gathered}
$$

and

$$
c_{*}\left[v_{\sharp}\right]\left(\bar{y}_{I}, \bar{s}\right)=c_{0}\left(\bar{y}_{I}\right)+\int_{\mathbb{R}^{N}} \mathcal{J}\left(y-\bar{y}_{I}\right)\left\{E_{*}\left(v_{\sharp}(y, \bar{s})-v_{\sharp}\left(\bar{y}_{I}, \bar{s}\right)\right)-p \cdot\left(y-\bar{y}_{I}\right)\right\} d y .
$$

By taking into account the auxiliary velocity $\tilde{c}_{*}$ defined in (4.5) and its Lipschitz constant $\mathcal{L}$ given by (4.6), it follows that

$$
\begin{aligned}
& c^{*}[u](\bar{x}, \bar{t})-c_{*}\left[v_{\sharp}\right]\left(\bar{y}_{I}, \bar{s}\right) \leq \tilde{c}_{*}\left[v_{\sharp}, \bar{y}_{I}\right](\bar{x}, \bar{s})-\tilde{c}_{*}\left[v_{\sharp}, \bar{y}_{I}\right]\left(\bar{y}_{I}, \bar{s}\right) \leq \\
\leq & \operatorname{Lip}\left(\tilde{c}_{*}\left[v_{\sharp}, \bar{y}_{I}\right](\cdot, \bar{s})\right)\left|\bar{x}-\bar{y}_{I}\right| \leq \mathcal{L}\left(\tilde{c}_{*}\left[v_{\sharp}\right]\right)(|\bar{x}-\bar{y}|+\sqrt{N} \Delta x / 2) .
\end{aligned}
$$

\section{Step 7: intermediate conclusion}

By (4.16),(4.17),(4.18) and the estimate (4.12) we obtain

$$
\eta<\frac{\Delta t}{2 \gamma}+\frac{1}{\gamma} \mathcal{L}\left(\tilde{c}_{*}\left[v_{\sharp}\right]\right)\left(2 \mathcal{G}^{\Delta x}\left(v_{\sharp}\right) \gamma+(1+\sqrt{N} / 2) \Delta x\right)^{2}+\left\|c_{*}\left[v_{\sharp}\right]\right\|_{\infty} \sqrt{\frac{N}{2}} \frac{\Delta x}{\gamma} .
$$

We set

$$
\begin{gathered}
K_{v_{\sharp}}^{I}=4(1+\sqrt{N} / 2) \mathcal{G}^{\Delta x}\left(v_{\sharp}\right) \mathcal{L}\left(\tilde{c}_{*}\left[v_{\sharp}\right]\right), \quad K_{v_{\sharp}}^{I I}=4\left(\mathcal{G}^{\Delta x}\left(v_{\sharp}\right)\right)^{2} \mathcal{L}\left(\tilde{c}_{*}\left[v_{\sharp}\right]\right), \\
K_{v_{\sharp}}^{I I I}=\left\|c_{*}\left[v_{\sharp}\right]\right\|_{\infty} \sqrt{\frac{N}{2}}, \quad K_{v_{\sharp}}^{I V}=(1+\sqrt{N} / 2)^{2} \mathcal{L}\left(\tilde{c}_{*}\left[v_{\sharp}\right]\right) .
\end{gathered}
$$

and we conclude that $\eta<\bar{\eta}$, with

$$
\bar{\eta}:=K_{v_{\sharp}}^{I} \Delta x+K_{v_{\sharp}}^{I I} \gamma+\frac{1}{\gamma}\left(\frac{\Delta t}{2}+K_{v_{\sharp}}^{I I I} \Delta x+K_{v_{\sharp}}^{I V} \Delta x^{2}\right) .
$$


If we choose $\eta \geq \bar{\eta}$, we obtain a contraddiction and then it is $\bar{t}=0$ or $\bar{s}=0$.

\section{Step 8: bound on the error}

In the case $\bar{t}=0$ we obtain

$$
\begin{gathered}
M=\max \Psi=\Psi(\bar{x}, 0, \bar{y}, \bar{s}) \leq u(\bar{x}, 0)-v_{\sharp}(\bar{y}, \bar{s}) \leq \\
\leq\left|u_{0}(\bar{x})-u_{0}(\bar{y})\right|+u_{0}(\bar{y})-\left(v_{0}\right)_{\sharp}(\bar{y})+\left(v_{0}\right)_{\sharp}(\bar{y})-v_{\sharp}(\bar{y}, \bar{s}) \leq \\
\leq\left\|\nabla u_{0}\right\|_{\infty}|\bar{x}-\bar{y}|+\mu_{0}+C_{v_{0}}|\bar{t}-\bar{s}| \leq \\
\leq\left\|\nabla u_{0}\right\|_{\infty}\left(2 \mathcal{G}^{\Delta x}\left(v_{\sharp}\right) \gamma+\Delta x\right)+C_{v_{0}}\left(4 \mathcal{G}^{\Delta t}\left(v_{\sharp}\right) \gamma+2 \Delta t\right)+\mu_{0}
\end{gathered}
$$

where we used (4.11) in the third line and (4.12),(4.13) in the fourth line.

Similarly, in the case $\bar{s}=0$ we obtain

$$
\begin{gathered}
M=\max \Psi=\Psi(\bar{x}, \bar{t}, \bar{y}, 0) \leq u(\bar{x}, \bar{t})-v_{\sharp}(\bar{y}, 0) \leq \\
\leq u(\bar{x}, \bar{t})-u(\bar{x}, 0)+\left|u_{0}(\bar{x})-u_{0}(\bar{y})\right|+u_{0}(\bar{y})-\left(v_{0}\right)_{\sharp}(\bar{y}) \leq \\
\leq C_{u_{0}}|\bar{t}-\bar{s}|+\left\|\nabla u_{0}\right\|_{\infty}|\bar{x}-\bar{y}|+\mu_{0} \leq \\
\leq C_{u_{0}}\left(4 \mathcal{G}^{\Delta t}\left(v_{\sharp}\right) \gamma+2 \Delta t\right)+\left\|\nabla u_{0}\right\|_{\infty}\left(2 \mathcal{G}^{\Delta x}\left(v_{\sharp}\right) \gamma+\Delta x\right)+\mu_{0} .
\end{gathered}
$$

where in the third line we used (4.10). Then from (4.21),(4.22) we get for some constants $K^{V}$ and $K^{V I}$

$$
M=\max \Psi \leq \mu_{0}+K^{V}(\Delta x+\Delta t)+K^{V I} \gamma,
$$

which, together with (4.20), implies that for every $x \in \mathbb{R}^{N}$ and $s \in\left\{0, \ldots, t_{n_{T}}\right\}$

$$
u(x, s)-v_{\sharp}(x, s)-\bar{\eta} s=\Psi(x, s, x, s) \leq M
$$

i.e.

$$
u(x, s)-v_{\sharp}(x, s) \leq M+\bar{\eta} T .
$$

By choosing

$$
\gamma=\sqrt{\frac{K_{v_{\sharp}}^{I I}}{K_{v_{\sharp}}^{I I}}\left(\Delta x+\frac{\Delta t}{2 K_{v_{\sharp}}^{I I I}}\right)}
$$

we get for some constant $K_{v_{\sharp}, u_{0}, v_{0}}$

$$
M \leq \mu_{0}+K_{v_{\sharp}, u_{0}, v_{0}}(\Delta x+\Delta t+\sqrt{\Delta x+\Delta t})
$$

and

$$
\bar{\eta}=K_{v_{\sharp}}^{I} \Delta x+2 \sqrt{K_{v_{\sharp}}^{I I} K_{v_{\sharp}}^{I I I}} \sqrt{\Delta x+\frac{\Delta t}{2 K_{v_{\sharp}}^{I I}}}+K_{v_{\sharp}}^{I V} \sqrt{\frac{K_{v_{\sharp}}^{I I}}{K_{v_{\sharp}}^{I I I}}} \sqrt{\frac{\Delta x}{\Delta x+\frac{\Delta t}{2 K_{v_{\sharp}}^{I I I}}}} \Delta x^{\frac{3}{2}} \leq \eta^{*},
$$

where $\eta^{*}$ is given by (4.8). Using (4.24) we conclude

$$
\sup _{\mathbb{R}^{N} \times\left\{0, \ldots, t_{n_{T}}\right\}}\left(u-v_{\sharp}\right) \leq \sup _{\mathbb{R}^{N}}\left(u_{0}-\left(v_{0}\right)_{\sharp}\right)+K_{v_{\sharp}, u_{0}, v_{0}}(\Delta x+\Delta t+\sqrt{\Delta x+\Delta t})+\eta^{*} T .
$$

In the case of general initial data we can replace $u$ with $\bar{u}=u+\mu_{1}$, where $\mu_{1}=\sup _{\mathbb{R}^{N}}\left(\left(v_{0}\right)_{\sharp}-u_{0}\right)$. Then $\bar{u}$ satisfies (4.9) and we have

$$
\sup _{\mathbb{R}^{N} \times\left\{0, \ldots, t_{n_{T}}\right\}}\left(u+\mu_{1}-v_{\sharp}\right) \leq \sup _{\mathbb{R}^{N}}\left(u_{0}+\mu_{1}-\left(v_{0}\right)_{\sharp}\right)+K_{v_{\sharp}, u_{0}, v_{0}}(\Delta x+\Delta t+\sqrt{\Delta x+\Delta t})+\eta^{*} T,
$$

which still implies (4.25). The proof is complete.

Remark 4.5 By exchanging the role of $u$ and $v_{\sharp}$ in Theorem 4.3 we can prove the same error estimate for $\sup \left(v_{\sharp}-u\right)$, with $v_{\sharp}$ sub-solution of the scheme (3.4), u a continuous super-solution of (3.1) and $c_{*}, \mathcal{L}\left(\tilde{c}_{*}\right)$ replaced by $c^{*}, \mathcal{L}\left(\tilde{c}^{*}\right)$ in the definition (4.8) of $\eta^{*}$. 


\subsection{Estimate for the cell problem}

Here we apply the error estimate (4.7) for the evolution problem and obtain an analogous error estimate for the effective Hamiltonian of the cell problem (1.6).

Theorem 4.6 (A posteriori $\mathcal{O}(\sqrt{\Delta x})$ error estimate)

For every $p \in \mathbb{R}^{N}$, let $\lambda$ be the effective Hamiltonian for the cell problem (1.6). For every discrete function $v_{0}$ satisfying (1.7), set $\bar{\lambda}^{d}=\sup _{I \in \mathbb{Z}^{N}}\left(R_{*}\right)_{I}\left[v_{0}\right]$ (resp. $\left.\underline{\lambda}^{d}=\inf _{I \in \mathbb{Z}^{N}} R_{I}^{*}\left[v_{0}\right]\right)$. Then the following error estimate holds:

$$
\begin{gathered}
\lambda-\bar{\lambda}^{d} \leq C_{N} \mathcal{G}_{v_{0}}^{\Delta x} c_{v_{0}} \mathbf{Y}(1+\mathbf{Y}(1+\sqrt{N} / 2))^{2} \\
\left(\text { resp. } \quad \underline{\lambda}^{d}-\lambda \leq C_{N} \mathcal{G}_{v_{0}}^{\Delta x} c_{v_{0}} \mathbf{Y}(1+\mathbf{Y}(1+\sqrt{N} / 2))^{2}\right),
\end{gathered}
$$

where

$$
\begin{aligned}
& C_{N}=2 \sqrt{2 N}, \quad \mathcal{G}_{v_{0}}^{\Delta x}=\sup _{|x-y| \geq \Delta x} \frac{\left|\left(v_{0}\right)_{\sharp}(x)-\left(v_{0}\right)_{\sharp}(y)\right|}{|x-y|}, \quad \mathbf{Y}=\sqrt{\frac{\mathcal{L}_{v_{0}} \Delta x}{c_{v_{0}} \sqrt{N / 2}}}, \\
& c_{v_{0}}=\left\|c_{*}\left[\left(v_{0}\right)_{\sharp}\right]\right\|_{\infty}, \quad \quad \mathcal{L}_{v_{0}}=\sup _{I \in \mathbb{Z}} \operatorname{Lip}\left(\tilde{c}_{*}\left[\left(v_{0}\right)_{\sharp}, x_{I}\right](\cdot)\right) \\
& \left(\text { resp. } \quad c_{v_{0}}=\left\|c^{*}\left[\left(v_{0}\right)_{\sharp}\right]\right\|_{\infty}, \quad \mathcal{L}_{v_{0}}=\sup _{I \in \mathbb{Z}} \operatorname{Lip}\left(\tilde{c}^{*}\left[\left(v_{0}\right)_{\sharp}, x_{I}\right](\cdot)\right)\right),
\end{aligned}
$$

with $\tilde{c}_{*}\left[\left(v_{0}\right)_{\sharp}, x_{I}\right]$ and $\tilde{c}^{*}\left[\left(v_{0}\right)_{\sharp}, x_{I}\right]$ defined in (4.5).

\section{Proof.}

Let $u$ be the unique continuous viscosity solution of the evolution problem (3.1) for some initial datum $u_{0}$. On the other hand define

$$
v_{I}^{n}=\left(v_{0}\right)_{I}+\bar{\lambda}^{d} n \Delta t, \quad I \in \mathbb{Z}^{N}, n \in \mathbb{N} .
$$

It follows that $v$ is a super-solution of the scheme (3.4) with initial datum $v_{0}$. We apply the error estimate (4.7) to $u$ and $v$ with $T=n \Delta t$ for every $x \in \mathbb{R}^{N}$ :

$$
u(x, T)-v_{\sharp}(x, T) \leq \sup _{\mathbb{R}^{N}}\left(u_{0}-\left(v_{0}\right)_{\sharp}\right)+K_{v_{\sharp}, u_{0}, v_{0}}(\Delta x+\Delta t+\sqrt{\Delta x+\Delta t})+\eta^{*} T .
$$

By following the proof of Theorem 4.3 and using the special form of $v$, it is easy to check that now $K_{v_{\sharp}, u_{0}, v_{0}}$ and $\eta^{*}$ do not depend on the whole $v_{\sharp}$, but only on $v_{0}$; in particular they do not depend on $T$. Then we can divide by $T$ and obtain

$$
\frac{u(x, T)}{T}-\frac{\left(v_{0}\right)_{\sharp}(x)}{T}-\bar{\lambda}^{d} \leq \frac{1}{T} \sup _{\mathbb{R}^{N}}\left(u_{0}-\left(v_{0}\right)_{\sharp}\right)+\frac{1}{T} K_{v_{\sharp}, u_{0}, v_{0}}(\Delta x+\Delta t+\sqrt{\Delta x+\Delta t})+\eta^{*} .
$$

By taking the limit as $T \rightarrow \infty$ and using the fact that $u(x, T) / T$ converges to $\lambda$ locally uniformly on $x$ (Theorem 3.3), we get

$$
\lambda-\bar{\lambda}^{d} \leq \eta^{*}
$$

and then, for $\Delta t \rightarrow 0$, we conclude to the result.

The estimate for $\underline{\lambda}^{d}$ can be obtained with the same argument by using Remark 4.5. The proof is complete.

Remark 4.7 The Lipschitz constant $\mathcal{L}_{v_{0}}$ can be estimated as follows:

$$
\mathcal{L}_{v_{0}} \leq \mathcal{L}_{v_{0}}^{*}:=\operatorname{Lip}\left(c_{0}\right)+|p|\|\mathcal{J}\|_{L^{1}\left(\mathbb{R}^{N}\right)}+\|\nabla \mathcal{J}\|_{L^{1}\left(\mathbb{R}^{N}\right)}\left(\frac{1}{2}+\operatorname{osc}\left(\left(v_{0}\right)_{\sharp}(y)-p \cdot y\right)\right),
$$

where the oscillation of a given function $w: \mathbb{R}^{N} \rightarrow \mathbb{R}$ is defined by

$$
\operatorname{osc}(w)=\sup _{x \in \mathbb{R}^{N}} w(x)-\inf _{x \in \mathbb{R}^{N}} w(x) .
$$

Now the proof of Theorem 1.3 is just a consequence of Theorem 4.6 and Remark 4.7. 


\section{Improved error estimate for the 1D case}

In this section we restrict to the one-dimensional setting and we improve, under further assumptions, the a posteriori error estimate given by Theorem 4.6. The idea is to use a discrete sub-solution of the scheme (3.4), to build, up to an error, a continuous sub-solution of the equation (3.1). This allows to obtain an error estimate for the effective Hamiltonian of order $\Delta x$, instead of $\sqrt{\Delta x}$.

\section{Theorem 5.1 (A posteriori $\mathcal{O}(\Delta x)$ error estimate)}

Let $N=1, p \in \mathbb{R}$ and let $\lambda$ be the effective Hamiltonian of the cell problem (1.6). For every discrete function $v$ satisfying (1.7), set $\underline{\lambda}^{d}=\inf _{i \in \mathbb{Z}} R_{i}^{*}[v]$ (resp. $\bar{\lambda}^{d}=\sup _{i \in \mathbb{Z}}\left(R_{*}\right)_{i}[v]$ ) and assume that

i) there exist constants $l_{0}, L_{0}$ such that $0<l_{0} \leq \frac{v_{i+1}-v_{i}}{\Delta x} \leq L_{0}$ for all $i \in \mathbb{Z}$;

ii) $\left(c^{*}\right)_{i}^{d}[v] \geq 0$ (resp. $\left.\left(c^{*}\right)_{i}^{d}[v] \geq 0\right)$ for all $i \in \mathbb{Z}$;

iii) $\left(1+\frac{L_{0}}{l_{0}}\right) \Delta x \leq \frac{1}{2 L_{0}}$;

iv) there exists a constant $C_{\mathcal{J}}$ such that the kernel $\mathcal{J}$ satisfies

$$
\mathcal{J}(z) \leq C_{\mathcal{J}} / z^{2} \quad \text { for all } z \in \mathbb{R} .
$$

Then the following error estimate holds:

$$
\underline{\lambda}^{d}-\lambda \leq K_{v} \Delta x, \quad\left(\text { resp. } \lambda-\bar{\lambda}^{d} \leq K_{v} \Delta x\right),
$$

where the constant $K_{v}$ is given by

$$
K_{v}=L_{0}\left\{\operatorname{Lip}\left(c_{0}\right)+\frac{4}{3} \pi^{2} C_{\mathcal{J}} L_{0}^{2}\left(1+\frac{L_{0}}{l_{0}}\right)\right\} .
$$

Remark 5.2 The last term in the right hand side of (5.2) can be regarded as the Lipschitz constant of the non-local part in the velocity $\left(c^{*}\right)^{d}[v]$. Moreover, by definition of $\underline{\lambda}^{d}$, it follows that

$$
\underline{\lambda}^{d}=\inf _{i \in \mathbb{Z}} R_{i}^{*}[v] \geq \inf _{i \in \mathbb{Z}}\left(\frac{v_{i+1}-v_{i}}{\Delta x}\right) \inf _{i \in \mathbb{Z}}\left(c^{*}\right)_{i}^{d}[v] .
$$

Then, if $\inf _{i \in \mathbb{Z}}\left(c^{*}\right)_{i}^{d}[v]>0$, we have the following interpretation for the relative error between $\lambda$ and $\underline{\lambda}^{d}$ :

$$
e_{r}:=\frac{\underline{\lambda}^{d}-\lambda}{\underline{\lambda}^{d}} \leq \frac{\sup \left(v_{x}\right)}{\inf \left(v_{x}\right)} \frac{\mathcal{L}}{\inf _{i \in \mathbb{Z}}\left(c^{*}\right)_{i}^{d}[v]} \Delta x
$$

with

$$
\sup \left(v_{x}\right)=\sup _{i \in \mathbb{Z}}\left(\frac{v_{i+1}-v_{i}}{\Delta x}\right), \quad \inf \left(v_{x}\right)=\inf _{i \in \mathbb{Z}}\left(\frac{v_{i+1}-v_{i}}{\Delta x}\right), \quad \mathcal{L}=\frac{K_{v}}{L_{0}},
$$

where $\mathcal{L}$ can be regarded as a kind of Lipschitz constant of the velocity $c^{*}$.

\section{Proof of Theorem 5.1.}

We restrict the proof to $\underline{\lambda}^{d}$, since it is the same for $\bar{\lambda}^{d}$. By definition of $R^{*}$ we have for all $i \in \mathbb{Z}$

$$
R_{i}^{*}[v]=\left(\frac{v_{i+1}-v_{i}}{\Delta x}\right)\left(c_{0}\left(x_{i}\right)+\int_{\mathbb{R}} \mathcal{J}(z)\left\{E^{*}\left(v_{\sharp}\left(x_{i}+z\right)-v_{\sharp}\left(x_{i}\right)\right)-p z\right\} d z\right),
$$

where we recall that $v_{\sharp}$ is the piecewise extension of $v$. We now consider the piecewise linear extension $\tilde{v}$ of $v$, satisfying $\tilde{v}\left(x_{i}\right)=v_{i}$ for each $i \in \mathbb{Z}$. It follows that, for all $i \in \mathbb{Z}$ and $x \in\left(x_{i}, x_{i+1}\right)$,

$$
\left|\tilde{v}_{x}\right| c^{*}[\tilde{v}](x)=\left(\frac{v_{i+1}-v_{i}}{\Delta x}\right)\left(c_{0}(x)+\int_{\mathbb{R}} \mathcal{J}(z)\left\{E^{*}(\tilde{v}(x+z)-\tilde{v}(x))-p z\right\} d z\right) .
$$


We want to compare $R^{*}[v]$ and $\left|\tilde{v}_{x}\right| c^{*}[\tilde{v}]$. To this end fix $x \in \mathbb{R}$ and choose $i \in \mathbb{Z}^{N}$ such that $x \in\left[x_{i}, x_{i+1}\right]$. Let $z_{j}=j \Delta x$, with $j \in \mathbb{Z}$, the minimal grid point such that

$$
E^{*}\left(v_{\sharp}\left(x_{i}+z_{j}\right)-v_{\sharp}\left(x_{i}\right)\right)=j+\frac{1}{2} .
$$

Similarly, let $z_{j}^{x} \in \mathbb{R}$ the minimal point such that

$$
E^{*}\left(\tilde{v}\left(x+z_{j}^{x}\right)-\tilde{v}(x)\right)=j+\frac{1}{2} .
$$

We remark that for $j=0$ we get $z_{0}=z_{0}^{x}=0$.

Step 1: $\left|z_{j}-z_{j}^{x}\right| \leq \Delta x\left(1+\frac{L_{0}}{l_{0}}\right)$.

We compare $z_{j}$ and $z_{j}^{x}$. By definition of $z_{j}$ we have

$$
v_{\sharp}\left(x_{i}+z_{j}\right)-v_{\sharp}\left(x_{i}\right) \geq j \quad \text { and } \quad v_{\sharp}\left(x_{i}+z_{j}-\Delta x\right)-v_{\sharp}\left(x_{i}\right)<j .
$$

Moreover by assumption $(i)$ we have

$$
0 \leq v_{\sharp}\left(x_{i}+z_{j}\right)-v_{\sharp}\left(x_{i}+z_{j}-\Delta x\right) \leq L_{0} \Delta x .
$$

Then it follows that

$$
j \leq v_{\sharp}\left(x_{i}+z_{j}\right)-v_{\sharp}\left(x_{i}\right) \leq v_{\sharp}\left(x_{i}+z_{j}-\Delta x\right)+L_{0} \Delta x-v_{\sharp}\left(x_{i}\right)<j+L_{0} \Delta x .
$$

On the other hand by definition of $z_{j}^{x}$ we have

$$
\tilde{v}\left(x+z_{j}^{x}\right)-\tilde{v}(x)=j
$$

which, together with (5.5), implies

$$
0 \leq v_{\sharp}\left(x_{i}+z_{j}\right)-v_{\sharp}\left(x_{i}\right)-\tilde{v}\left(x+z_{j}^{x}\right)+\tilde{v}(x)<L_{0} \Delta x .
$$

By construction $\tilde{v}$ coincides with $v_{\sharp}$ on the grid and by assumption $(i)$ we have

$$
-L_{0} \Delta x \leq \tilde{v}\left(x_{i}\right)-\tilde{v}(x) \leq 0 .
$$

Then we get

$$
\left|\tilde{v}\left(x_{i}+z_{j}\right)-\tilde{v}\left(x+z_{j}^{x}\right)\right| \leq L_{0} \Delta x .
$$

Moreover

$$
\left|\tilde{v}\left(x_{i}+z_{j}\right)-\tilde{v}\left(x+z_{j}^{x}\right)\right| \geq l_{0}\left|x_{i}+z_{j}-x-z_{j}^{x}\right| \geq l_{0}\left(\left|z_{j}-z_{j}^{x}\right|-\left|x-x_{i}\right|\right)
$$

and then we conclude

$$
\left|z_{j}-z_{j}^{x}\right| \leq \Delta x\left(1+\frac{L_{0}}{l_{0}}\right) .
$$

Step 2: bound from below on $\left|z_{j}^{x}\right|$.

Consider for all $j \in \mathbb{Z}$ the points $z_{j}^{x}$ and $z_{j+1}^{x}$ : by definition

$$
\tilde{v}\left(x+z_{j+1}^{x}\right)-\tilde{v}(x)=j+1 \quad \text { and } \quad \tilde{v}\left(x+z_{j}^{x}\right)-\tilde{v}(x)=j .
$$

By assumption $(i)$ we get

$$
1=\tilde{v}\left(x+z_{j+1}^{x}\right)-\tilde{v}\left(x+z_{j}^{x}\right) \leq L_{0}\left(z_{j+1}^{x}-z_{j}^{x}\right)
$$

and then

$$
z_{j+1}^{x}-z_{j}^{x} \geq \frac{1}{L_{0}},
$$

which implies

$$
\left|z_{j}^{x}\right| \geq \frac{|j|}{L_{0}}
$$


Step 3: comparison of $R^{*}[v]$ and $\left|\tilde{v}_{x}\right| c^{*}[\tilde{v}]$.

We set

$$
m_{j}=\min \left\{z_{j}, z_{j}^{x}\right\} \quad \text { and } \quad M_{j}=\max \left\{z_{j}, z_{j}^{x}\right\} .
$$

We remark that, by (5.7), (5.8) and assumption (iii), the intervals $\left(m_{j}, M_{j}\right)$ are disjoint. It follows that for any $z \in \mathbb{R}$

$$
\left|E^{*}\left(v_{\sharp}\left(x_{i}+z\right)-v_{\sharp}\left(x_{i}\right)\right)-E^{*}(\tilde{v}(x+z)-\tilde{v}(x))\right|=\sum_{j \in \mathbb{Z} \backslash\{0\}} \chi_{\left(m_{j}, M_{j}\right)}(z),
$$

where the index $j=0$ is excluded from the sum, since $m_{0}=M_{0}=z_{0}=z_{0}^{x}=0$. Then, by using the fact that the kernel $\mathcal{J}$ is an even non-negative function and assumption $(i v)$, we obtain for any $x \in\left[x_{i}, x_{i+1}\right]$

$$
\begin{aligned}
\mid R_{i}^{*}[v] & -\left(\frac{v_{i+1}-v_{i}}{\Delta x}\right) c^{*}[\tilde{v}](x)\left|=\left(\frac{v_{i+1}-v_{i}}{\Delta x}\right)\right|\left(c^{*}\right)_{i}^{d}-c^{*}[\tilde{v}](x) \mid \leq \\
& \leq\left(\frac{v_{i+1}-v_{i}}{\Delta x}\right)\left|c_{0}\left(x_{i}\right)-c_{0}(x)+2 C_{\mathcal{J}} \sum_{j \geq 1} \int_{m_{j}}^{M_{j}} \frac{d z}{z^{2}}\right| .
\end{aligned}
$$

Moreover, by (5.7), (5.9) and assumption (iii), we get

$$
M_{j}-m_{j} \leq\left(1+\frac{L_{0}}{l_{0}}\right) \Delta x \quad \text { and } \quad m_{j} \geq \frac{j}{L_{0}}-\left(1+\frac{L_{0}}{l_{0}}\right) \Delta x \geq \frac{j}{2 L_{0}},
$$

which implies

$$
\sum_{j \geq 1} \int_{m_{j}}^{M_{j}} \frac{d z}{z^{2}} \leq \sum_{j \geq 1} \int_{j / 2 L_{0}}^{j / 2 L_{0}+\left(1+L_{0} / l_{0}\right) \Delta x} \frac{d z}{z^{2}} \leq\left(2 L_{0}\right)^{2}\left(1+\frac{L_{0}}{l_{0}}\right) \Delta x \sum_{j \geq 1} \frac{1}{j^{2}}=\frac{2}{3} \pi^{2} L_{0}^{2}\left(1+\frac{L_{0}}{l_{0}}\right) \Delta x,
$$

where we used the identity $\sum_{j \geq 1}^{\infty} 1 / j^{2}=\pi^{2} / 6$. From (5.10) we conclude

$$
\left|R^{*}[v]_{i}-\left(\frac{v_{i+1}-v_{i}}{\Delta x}\right) c^{*}[\tilde{v}](x)\right| \leq L_{0}\left\{\operatorname{Lip}\left(c_{0}\right)+\frac{4}{3} \pi^{2} C_{\mathcal{J}} L_{0}^{2}\left(1+\frac{L_{0}}{l_{0}}\right)\right\} \Delta x=: e .
$$

By definition of $\underline{\lambda}^{d}$ it follows that for any $i \in \mathbb{Z}$ and $x \in\left[x_{i}, x_{i+1}\right]$

$$
\underline{\lambda}^{d}=\inf _{i \in \mathbb{Z}} R^{*}[v]_{i} \leq R^{*}[v]_{i} \leq\left(\frac{v_{i+1}-v_{i}}{\Delta x}\right) c^{*}[\tilde{v}](x)+e .
$$

We want to prove that inequality (5.12) implies

$$
\underline{\lambda}^{d} \leq\left|\tilde{v}_{x}\right| c^{*}[\tilde{v}](x)+e .
$$

in the viscosity sense for any $x \in \mathbb{R}$. To this end it is enough to show that for every end-point $x_{i}$ we also have

$$
\underline{\lambda}^{d} \leq\left(\frac{v_{i}-v_{i-1}}{\Delta x}\right) c^{*}[\tilde{v}]\left(x_{i}\right)+e .
$$

This easily follows by applying (5.12) in the interval $\left[x_{i-1}, x_{i}\right]$ for $x=x_{i}$ :

$$
\underline{\lambda}^{d}=\inf _{i \in \mathbb{Z}} R^{*}[v]_{i} \leq R^{*}[v]_{i-1} \leq\left(\frac{v_{(i-1)+1}-v_{(i-1)}}{\Delta x}\right) c^{*}[\tilde{v}]\left(x_{i}\right)+e=\left(\frac{v_{i}-v_{i-1}}{\Delta x}\right) c^{*}[\tilde{v}]\left(x_{i}\right)+e .
$$

Step 4: conclusion.

Define for $x \in \mathbb{R}$ and $t \geq 0$

$$
\tilde{u}(t, x)=\left(\underline{\lambda}^{d}-e\right) t+\tilde{v}(x) .
$$

It follows that $\tilde{u}$ is a sub-solution of the evolution problem (3.1) with initial datum $\tilde{v}$. Indeed, by (5.13) and the invariance with respect to the addition of constants, we get in the viscosity sense

$$
u_{t}(t, x)=\underline{\lambda}^{d}-e \leq\left|\tilde{v}_{x}\right| c^{*}[\tilde{v}](x)=\left|\tilde{u}_{x}(t, x)\right| c^{*}[\tilde{u}(t, \cdot)](x) .
$$


On the other hand let $U(x)$ be a corrector associated to the effective Hamiltonian $\lambda$, namely a viscosity solution of the cell problem

$$
\lambda=|\nabla U| c[U] \text { on } \quad \mathbb{R}^{N} .
$$

Up to add a constant to $U$, we can assume that $\tilde{v} \leq U$. It follows that the function

$$
u(t, x)=\lambda t+U(x)
$$

is a viscosity solution of (3.1) with inital datum $U(x)$ and then, by comparison between $\tilde{u}$ and $u$ (Theorem 3.2 ), we obtain

$$
\left(\underline{\lambda}^{d}-e\right) t+\tilde{v}(x) \leq \lambda t+U(x) .
$$

Dividing by $t$ and taking the limit as $t \rightarrow \infty$, we get

$$
\underline{\lambda}^{d}-\lambda \leq e
$$

and this finishes the proof.

We conclude this section with the proof of Theorem 1.4, which is a corollary of the previous result.

\section{Proof of Theorem 1.4.}

By assumptions (i)-(ii) of Theorem 1.4 the gradient of $v$ is strictly positive, i.e. $\frac{v_{i+1}-v_{i}}{\Delta x}>0$ for all $i \in \mathbb{Z}$, and we have

$$
0 \leq \underline{\lambda}^{d}=\inf _{i \in \mathbb{Z}} R_{i}^{*}[v] \leq R_{i}^{*}[v]= \begin{cases}\frac{v_{i+1}-v_{i}}{\Delta x}\left(c^{*}\right)_{i}^{d}[v] & \text { if }\left(c^{*}\right)_{i}^{d}[v] \geq 0 \\ \frac{v_{i}-v_{i-1}}{\Delta x}\left(c^{*}\right)_{i}^{d}[v] & \text { if }\left(c^{*}\right)_{i}^{d}[v]<0 .\end{cases}
$$

It follows that $\left(c^{*}\right)_{i}^{d}[v]$ is non-negative for all $i \in \mathbb{Z}$ and we conclude by applying Theorem 5.1.

\section{Discrete time evolution problem}

\subsection{Existence of discrete solutions}

In this section we prove an existence result of discrete solutions for the evolution scheme (3.4). To proceed let us fix some notations: for every $s \in \mathbb{R}$ and every discrete function $v$ we set

$$
\begin{gathered}
D_{k, v_{I}}^{+}(s)=\frac{v_{I+e_{k}}-s}{\Delta x}, \quad D_{k, v_{I}}^{-}(s)=\frac{s-v_{I-e_{k}}}{\Delta x}, \quad k=1, \ldots, N, \\
D_{v_{I}}(s)=\left(D_{v_{I}}^{+}(s), D_{v_{I}}^{-}(s)\right) \quad \text { with } \quad D_{v_{I}}^{ \pm}(s)=\left(D_{1, v_{I}}^{ \pm}(s), \ldots, D_{N, v_{I}}^{ \pm}(s)\right) .
\end{gathered}
$$

We also set, for every $I \in \mathbb{Z}^{N}$,

$$
\begin{gathered}
\left(c^{*}\right)_{I}^{d}[v](s)=c_{0}\left(x_{I}\right)+\sum_{J \in \mathbb{Z}^{N}} \int_{Q_{J}} \mathcal{J}(z)\left\{E^{*}\left(v_{\sharp}\left(x_{I}+z\right)-s\right)-p \cdot z\right\} d z, \\
\left(c_{*}\right)_{I}^{d}[v](s)=c_{0}\left(x_{I}\right)+\sum_{J \in \mathbb{Z}^{N}} \int_{Q_{J}} \mathcal{J}(z)\left\{E_{*}\left(v_{\sharp}\left(x_{I}+z\right)-s\right)-p \cdot z\right\} d z, \\
G_{\left(c^{*}\right)_{I}^{d}}[v](s)= \begin{cases}G^{+}\left(D_{v_{I}}(s)\right) & \text { if }\left(c^{*}\right)_{I}^{d}[v](s) \geq 0 \\
G^{-}\left(D_{v_{I}}(s)\right) & \text { if }\left(c^{*}\right)_{I}^{d}[v](s)<0,\end{cases} \\
G_{\left(c_{*}\right)_{I}^{d}}[v](s)= \begin{cases}G^{+}\left(D_{v_{I}}(s)\right) & \text { if }\left(c_{*}\right)_{I}^{d}[v](s) \geq 0 \\
G^{-}\left(D_{v_{I}}(s)\right) & \text { if }\left(c_{*}\right)_{I}^{d}[v](s)<0\end{cases}
\end{gathered}
$$

and

$$
\begin{gathered}
R_{I}^{*}[v](s)=G_{\left(c^{*}\right)_{I}^{d}}[v](s)\left(c^{*}\right)_{I}^{d}[v](s), \\
\left(R_{*}\right)_{I}[v](s)=G_{\left(c_{*}\right)_{I}^{d}}[v](s)\left(c_{*}\right)_{I}^{d}[v](s) .
\end{gathered}
$$


Remark 6.1 It easily follows by definition that, for every $I \in \mathbb{Z}^{N}$, the operators $R_{I}^{*}[v](s)$ and $\left(R_{*}\right)_{I}[v](s)$ satisfy the following properties:

a) $R_{I}^{*}[v](s)$ and $\left(R_{*}\right)_{I}[v](s)$ are non increasing with respect to $s$;

b) $R_{I}^{*}[v](s)$ and $\left(R_{*}\right)_{I}[v](s)$ are non decreasing with respect to $v$;

c) $R_{I}^{*}[v]\left(v_{I}\right)=R_{I}^{*}[v]$ and $\left(R_{*}\right)_{I}[v]\left(v_{I}\right)=\left(R_{*}\right)_{I}[v]$;

d) $\left(R_{*}\right)_{I}[v](s) \geq R_{I}^{*}[v](t)$ for all $s<t$.

We have the following lemma.

\section{Lemma 6.2 (Comparison)}

Let $v^{0}, v$ be discrete functions satisfying (1.7) and fix $I \in \mathbb{Z}^{N}$. There exists a unique $s_{I} \in \mathbb{R}$ such that

$$
\left(R_{*}\right)_{I}[v]\left(s_{I}\right) \leq \frac{s_{I}-v_{I}^{0}}{\Delta t} \leq R_{I}^{*}[v]\left(s_{I}\right)
$$

Moreover, let $w$ be a discrete functions satisfying (1.7). Then the following implications hold:

$$
\begin{aligned}
& \text { i) } \frac{w_{I}-v_{I}^{0}}{\Delta t} \leq R_{I}^{*}[w] \quad \text { and } w \leq v \quad \Longrightarrow \quad w_{I} \leq s_{I} \\
& \text { ii) } \frac{w_{I}-v_{I}^{0}}{\Delta t}>R_{I}^{*}[w] \quad \text { and } w \geq v \quad \Longrightarrow \quad w_{I}>s_{I} \\
& \text { iii) } \frac{w_{I}-v_{I}^{0}}{\Delta t} \geq\left(R_{*}\right)_{I}[w] \text { and } w \geq v \quad \Longrightarrow \quad w_{I} \geq s_{I} \\
& \text { iv) } \frac{w_{I}-v_{I}^{0}}{\Delta t}<\left(R_{*}\right)_{I}[w] \text { and } w \leq v \quad \Longrightarrow \quad w_{I}<s_{I}
\end{aligned}
$$

Proof.

The existence of a unique $s_{I} \in \mathbb{R}$ satisfying (6.1) immediately follows by the fact that $\left(R_{*}\right)_{I}[v](s), R_{I}^{*}[v](s)$ are non-increasing with respect to $s$ (see Remark 6.1) and the function $\frac{s-v_{I}^{0}}{\Delta t}$ is strictly increasing in $s$.

Now we prove implication $(i)$. By contradiction suppose that $w_{I}>s_{I}$. Then, by (6.1) and the properties in Remark 6.1, we get

$$
\frac{w_{I}-v_{I}^{0}}{\Delta t}>\frac{s_{I}-v_{I}^{0}}{\Delta t} \stackrel{(6.1)}{\geq}\left(R_{*}\right)_{I}[v]\left(s_{I}\right) \stackrel{w \leq v,(b)}{\geq}\left(R_{*}\right)_{I}[w]\left(s_{I}\right) \stackrel{(d)}{\geq} R_{I}^{*}[w]\left(w_{I}\right) \stackrel{(c)}{=} R_{I}^{*}[w] \geq \frac{w_{I}-v_{I}^{0}}{\Delta t},
$$

which is absurd. Similarly we get implication $(i i)$. Indeed, if we assume $w_{I} \leq s_{I}$, we obtain

$$
\frac{w_{I}-v_{I}^{0}}{\Delta t} \leq \frac{s_{I}-v_{I}^{0}}{\Delta t} \stackrel{(6.1)}{\leq} R_{I}^{*}[v]\left(s_{I}\right) \stackrel{w \geq v,(b)}{\leq} R_{I}^{*}[w]\left(s_{I}\right) \stackrel{(a)}{\leq} R_{I}^{*}[w]\left(w_{I}\right) \stackrel{(c)}{=} R_{I}^{*}[w] \stackrel{\downarrow}{<} \frac{w_{I}-v_{I}^{0}}{\Delta t} .
$$

Finally, implications $(i i i),(i v)$ follow by the same arguments. The proof is complete.

Now we can prove the announced existence result.

\section{Theorem 6.3 (Existence of discrete solutions)}

Let $u^{0}$ be a discrete function satisfying (1.7). There exists at least one solution of the scheme (3.4) with initial datum $u^{0}$.

\section{Proof.}

We first consider the discrete barriers given by Proposition 4.2, namely

$$
u_{I}^{ \pm, n}=u_{I}^{0} \pm C_{u^{0}} n \Delta t, \quad \text { for all } I \in \mathbb{Z}^{N}, n \in \mathbb{N}, \quad \text { with } \quad C_{u^{0}} \geq \max \left\{\left\|R_{*}\left[u^{0}\right]\right\|_{\infty},\left\|R^{*}\left[u^{0}\right]\right\|_{\infty}\right\},
$$


and we recall that every solution $u$ of the scheme (3.4) satisfies

$$
u_{I}^{-, n} \leq u_{I}^{n} \leq u_{I}^{+, n} \quad \text { for all } I \in \mathbb{Z}^{N}, n \in \mathbb{N} .
$$

Now we proceed by recurrence in time, i.e., we fix $n \in \mathbb{N}$, we assume that there exists a solution $u^{n}$ at step $n$ and we prove the existence of a solution $u^{n+1}$ at step $n+1$ by Perron's method. To this end we remark that $u^{-, n+1}$ and $u^{+, n+1}$ are respectively a sub-solution and a super-solution of the scheme at step $n+1$. Indeed, by (6.2) and the invariance of $R^{*}, R_{*}$ with respect to the addition of constants, we get for all $I \in \mathbb{Z}^{N}$

$$
\frac{u_{I}^{-, n+1}-u_{I}^{n}}{\Delta t} \leq \frac{u_{I}^{-, n+1}-u_{I}^{-, n}}{\Delta t}=-C_{u^{0}} \leq R_{I}^{*}\left[u^{0}\right]=R_{I}^{*}\left[u^{-, n+1}\right]
$$

and

$$
\frac{u_{I}^{+, n+1}-u_{I}^{n}}{\Delta t} \geq \frac{u_{I}^{+, n+1}-u_{I}^{+, n}}{\Delta t}=C_{u^{0}} \geq\left(R_{*}\right)_{I}\left[u^{0}\right]=\left(R_{*}\right)_{I}\left[u^{+, n+1}\right] .
$$

Then we consider the following set:

$$
S:=\left\{w \text { sub-solution at step } n+1 \text { s.t. } w \leq u^{+, n+1}\right\}
$$

and define pointwise

$$
v:=\sup S .
$$

Note that $u^{-, n+1} \in S$ and then the function $v$ is well defined. If we prove that $v$ is both a sub-solution and a super-solution at step $n+1$, we conclude by setting $u^{n+1}:=v$. The proof is splitted in two steps.

Step 1: $v$ is a sub-solution of the scheme at time step $n+1$.

Let us fix $I \in \mathbb{Z}^{N}$ and consider, for $k=1, \ldots, N$, sequences $\left\{v^{m}\right\},\left\{\bar{v}^{k, m}\right\},\left\{\underline{v}^{k, m}\right\}$ in $S$ such that

$$
v_{I}^{m} \stackrel{m \rightarrow \infty}{\longrightarrow} v_{I}, \quad \bar{v}_{I+e_{k}}^{k, m} \stackrel{m \rightarrow \infty}{\longrightarrow} v_{I+e_{k}} \quad \underline{v}_{I-e_{k}}^{k, m} \stackrel{m \rightarrow \infty}{\longrightarrow} v_{I-e_{k}} .
$$

We then take the pointwise maximum between these $2 N+1$ sequences, that we denote again, with a little abuse of notation, by $v^{m}$ so that

$$
v_{J}^{m} \leq v_{J} \quad \forall J \in \mathbb{Z}^{N}, \quad v_{I}^{m} \stackrel{m \rightarrow \infty}{\longrightarrow} v_{I}, \quad v_{I \pm e_{k}}^{m} \stackrel{m \rightarrow \infty}{\longrightarrow} v_{I \pm e_{k}} \quad \text { for } k=1, \ldots, N
$$

and we remark that, by Lemma $2.6, v^{m}$ is still a sequence of sub-solutions.

For every $J \in \mathbb{Z}^{N}$ we have

$$
\limsup _{m \rightarrow \infty}\left(v_{I+J}^{m}-v_{I}^{m}\right) \leq v_{I+J}-v_{I} .
$$

Let $l \in \mathbb{Z}$ such that $l-1<v_{I+J}-v_{I}<l+1$. By (6.3) we get $v_{I+J}^{m}-v_{I}^{m}<l+1$ for $m$ large enough. Then in both cases $v_{I+J}-v_{I} \geq l$ and $v_{I+J}-v_{I}<l$ we get

$$
E^{*}\left(v_{I+J}^{m}-v_{I}^{m}\right)=E^{*}\left(v_{I+J}-v_{I}\right)
$$

or

$$
E^{*}\left(v_{I+J}^{m}-v_{I}^{m}\right)<E^{*}\left(v_{I+J}-v_{I}\right) .
$$

This implies $\left(c^{*}\right)_{I}^{d}\left[v^{m}\right] \leq\left(c^{*}\right)_{I}^{d}[v]$ for $m$ large enough.

On the other hand we have

$$
D_{k}^{+} v_{I}^{m}=\frac{v_{I+e_{k}}^{m}-v_{I}^{m}}{\Delta x} \stackrel{m \rightarrow \infty}{\longrightarrow} D_{k}^{+} v_{I}, \quad D_{k}^{-} v_{I}^{m}=\frac{v_{I}^{m}-v_{I-e_{k}}^{m}}{\Delta x} \stackrel{m \rightarrow \infty}{\longrightarrow} D_{k}^{-} v_{I}, \quad k=1, \ldots, N
$$

and

$$
G^{ \pm}\left(D^{+} v_{I}^{m}, D^{-} v_{I}^{m}\right) \stackrel{m \rightarrow \infty}{\longrightarrow} G^{ \pm}\left(D^{+} v_{I}, D^{-} v_{I}\right) .
$$

By reasoning as in the proof of Lemma 2.6, depending on the signs of $\left(c^{*}\right)_{I}^{d}\left[v^{m}\right]$ and $\left(c^{*}\right)_{I}^{d}[v]$, we conclude that

$$
\limsup _{m \rightarrow \infty} R_{I}^{*}\left[v^{m}\right] \leq R_{I}^{*}[v]
$$


But for every $m$ the function $v^{m}$ is a sub-solution of the scheme at step $n+1$ :

$$
v_{I}^{m} \leq u_{I}^{n}+\Delta t R_{I}^{*}\left[v^{m}\right]
$$

By taking the limit as $m$ goes to infinity we finally get

$$
v_{I} \leq u_{I}^{n}+\Delta t \limsup _{m \rightarrow \infty} R_{I}^{*}\left[v^{m}\right] \leq u_{I}^{n}+\Delta t R_{I}^{*}[v] .
$$

Since $I \in \mathbb{Z}^{N}$ is arbitrary we conclude that $v=\sup S$ is still a sub-solution of the scheme at step $n+1$.

Step 2: $v$ is a super-solution of the scheme at time step $n+1$.

By contradiction we assume that the super-solution inequality fails at least at one point, namely

$$
v_{I_{0}}<u_{I_{0}}^{n}+\Delta t\left(R_{*}\right)_{I_{0}}[v] \quad \text { for some } I_{0} \in \mathbb{Z}^{N} .
$$

By Lemma 6.2 there exists a unique $s_{I_{0}} \in \mathbb{R}$ such that

$$
\left(R_{*}\right)_{I_{0}}[v]\left(s_{I_{0}}\right) \leq \frac{s_{I_{0}}-u_{I_{0}}^{n}}{\Delta t} \leq R_{I_{0}}^{*}[v]\left(s_{I_{0}}\right) \quad \text { and } \quad v_{I_{0}}<s_{I_{0}} \leq u_{I_{0}}^{+, n+1}
$$

Then we define a new discrete function $w$ as follows:

$$
w_{I}= \begin{cases}s_{I_{0}} & \text { if } I=I_{0}, \\ v_{I} & \text { otherwise } .\end{cases}
$$

By (6.4) and monotonicity properties of $R^{*}$ (see Remark 6.1), we obtain

$$
w_{I_{0}} \leq u_{I_{0}}^{n}+\Delta t R_{I_{0}}^{*}[v]\left(w_{I_{0}}\right) \leq u_{I_{0}}^{n}+\Delta t R_{I_{0}}^{*}[w],
$$

and we remark that the same inequality holds for $I \neq I_{0}$ by Lemma 2.6. Then $w$ is a sub-solution of the scheme at step $n+1$ such that $w \leq u^{+, n+1}$, i.e., $w \in S$ and it is greater than $v$ at one point. This contradicts the definition of $v$ as the supremum of $S$. Therefore $v$ is a super-solution and the proof is complete.

Remark 6.4 Since every solution of the scheme is bounded from above by the barrier $u^{+}$, it follows that the solution given by the Perron's method is the maximal solution of the scheme. By a similar proof we can prove that the function $v:=\inf I$, with

$$
I:=\left\{w \text { super-solution at step } n+1 \text { s.t. } w \geq u^{-, n+1}\right\},
$$

is the minimal solution of the scheme.

\subsection{Construction of minimal and maximal solutions}

This section is devoted to the proof of Theorem 1.5, i.e., we construct minimal and maximal solutions for the scheme (3.4). Moreover, we prove the monotonicity of the time derivative of a particular solution and use this information to improve the approximation of the effective Hamiltonian.

We start by showing how to produce a solution for a single time step $\Delta t$, given an initial datum and a sub-solution (or a super-solution), then we extend the results at each time step by recurrence. The main tool is the following

\section{Proposition 6.5 (Definition of the map $\Phi$ )}

There exists a map $\Phi: \mathbb{R}^{\mathbb{Z}^{N}} \times \mathbb{R}^{\mathbb{Z}^{N}} \rightarrow \mathbb{R}^{\mathbb{Z}^{N}}$ satisfying the following properties:

a) Consider discrete functions $u^{0}$ and $u^{-}$such that $u^{-}$is a sub-solution of the scheme (3.4) at step $\Delta t$ with initial datum $u^{0}$, i.e.,

$$
\frac{u_{I}^{-}-u_{I}^{0}}{\Delta t} \leq R_{I}^{*}\left[u^{-}\right], \quad I \in \mathbb{Z}^{N}
$$

Then

$$
\text { i) } u^{-} \leq \Phi\left[u^{0}, u^{-}\right] \quad \text { ( with } u^{-}=\Phi\left[u^{0}, u^{-}\right] \text {if and only if } u^{-} \text {is a solution ) }
$$


ii) $\Phi\left[u^{0}, u^{-}\right]$is a sub-solution

b) Consider discrete functions $v^{0}$ and $v^{+}$such that $v^{+}$is a super-solution of the scheme (3.4) at step $\Delta t$ with initial datum $v^{0}$, i.e.,

$$
\frac{v_{I}^{+}-v_{I}^{0}}{\Delta t} \geq\left(R_{*}\right)_{I}\left[v^{+}\right], \quad I \in \mathbb{Z}^{N} .
$$

Then

$$
\begin{aligned}
& \text { i) } v^{+} \geq \Phi\left[v^{0}, v^{+}\right] \quad \text { ( with } v^{+}=\Phi\left[v^{0}, v^{+}\right] \text {if and only if } v^{+} \text {is a solution ) } \\
& \text { ii) } \Phi\left[v^{0}, v^{+}\right] \text {is a super-solution }
\end{aligned}
$$

\section{Proof.}

We restrict the proof to properties $(a)$ for sub-solutions, since properties $(b)$ can be obtained by similar arguments.

We apply Lemma 6.1 to $u^{0}$ and $u^{-}$: for every $I \in \mathbb{Z}^{N}$ there exists a unique $s_{I}^{-}$such that

$$
\left(R_{*}\right)_{I}\left[u^{-}\right]\left(s_{I}^{-}\right) \leq \frac{s_{I}^{-}-u_{I}^{0}}{\Delta t} \leq R_{I}^{*}\left[u^{-}\right]\left(s_{I}^{-}\right) \quad \text { and } \quad u_{I}^{-} \leq s_{I}^{-} .
$$

Then we glue together all the values $s_{I}^{-}$and define the map $\Phi$, depending on the initial datum $u_{0}$ and the sub-solution $u^{-}$:

$$
\Phi_{I}\left[u^{0}, u^{-}\right]:=s_{I}^{-}, \quad I \in \mathbb{Z}^{N} .
$$

By construction we have $u^{-} \leq \Phi\left[u^{0}, u^{-}\right]$, with equality if and only if $u^{-}$is a solution, i.e., property $(i)$ is proved. Now consider, for fixed $J \in \mathbb{Z}^{N}$, the following function, obtained by changing the value of $u^{-}$at the point $J$ :

$$
u_{I}^{-, J}= \begin{cases}s_{J}^{-} & \text {if } I=J \\ u_{I}^{-} & \text {otherwise }\end{cases}
$$

By Lemma 2.6 it follows that $u^{-, J}$ is a sub-solution for all $J \in \mathbb{Z}^{N}$ and then $\Phi\left[u^{0}, u^{-}\right]$can be regarded as a pointwise supremum of sub-solutions, i.e.,

$$
\Phi_{I}\left[u^{0}, u^{-}\right]=\sup _{J \in \mathbb{Z}^{N}}\left\{u_{I}^{-, J}\right\} .
$$

By reasoning as in the proof of Theorem 6.3 we conclude that $\Phi\left[u^{0}, u^{-}\right]$is also a sub-solution, namely property (ii), and this finishes the proof.

Remark 6.6 The key point in our construction is that the map $\Phi$ strictly increases sub-solutions in all the points where they are not super-solutions and strictly decreases super-solutions in all the points where they are not sub-solutions.

The next result shows that the map $\Phi$ enjoys a kind of comparison principle.

\section{Proposition 6.7 (Partial comparison principle)}

Let $u^{0}, v^{0}$ be discrete functions such that $u^{0} \leq v^{0}$. Consider a sub-solution $u^{-}$at step $\Delta t$ with initial datum $u^{0}$, a super-solution $v^{+}$with initial datum $v^{0}$ and assume $u^{-} \leq v^{+}$. Then $\Phi\left[u^{0}, u^{-}\right] \leq \Phi\left[v^{0}, v^{+}\right]$.

\section{Proof.}

Suppose by contraddiction that there exists $I \in \mathbb{Z}^{N}$ such that

$$
\Phi_{I}\left[u^{0}, u^{-}\right]=s_{I}^{-}>s_{I}^{+}=\Phi_{I}\left[v^{0}, v^{+}\right] .
$$

By property $(d)$ in Remark 6.1, we have

$$
\left(R_{*}\right)_{I}[\cdot]\left(t_{1}\right) \geq R_{I}^{*}[\cdot]\left(t_{2}\right) \quad \forall t_{2}>t_{1}
$$

and this, together with $u^{0} \leq v^{0}, u^{-} \leq v^{+}$and monotonicity of $R^{*}, R_{*}$ implies an absurd:

$$
\begin{gathered}
u_{I}^{0}+\Delta t R_{I}^{*}\left[u^{-}\right]\left(s_{I}^{-}\right) \geq s_{I}^{-}>s_{I}^{+} \geq v_{I}^{0}+\Delta t\left(R_{*}\right)_{I}\left[v^{+}\right]\left(s_{I}^{+}\right) \geq \\
\quad \geq v_{I}^{0}+\Delta t\left(R_{*}\right)_{I}\left[u^{-}\right]\left(s_{I}^{+}\right) \geq u_{I}^{0}+\Delta t R_{I}^{*}\left[u^{-}\right]\left(s_{I}^{-}\right) .
\end{gathered}
$$

Now we can make a step in time and construct solutions. The idea is just to apply iteratively to $u^{-}, v^{+}$the map $\Phi$ and the partial comparison principle. We have the following result. 


\section{Proposition 6.8 (Construction of solutions)}

Under the assumptions of Proposition 6.7, set for every $k \in \mathbb{N}$

$$
u^{-, k+1}=\Phi\left[u^{0}, u^{-, k}\right] \quad \text { with } \quad u^{-, 0}=u^{-}
$$

and

$$
v^{+, k+1}=\Phi\left[v^{0}, v^{+, k}\right] \quad \text { with } \quad v^{+, 0}=v^{+} .
$$

There exist discrete functions $u^{1}$ and $v^{1}$ such that $u^{-, k} \rightarrow u^{1}$ and $v^{+, k} \rightarrow v^{1}$ as $k \rightarrow+\infty$. Moreover $u^{1}, v^{1}$ are solutions of the scheme (3.4) at step $\Delta t$ with initial data $u^{0}, v^{0}$ respectively. We set

$$
\Psi\left[u^{0}, u^{-}\right]:=u^{1} \quad \text { and } \quad \Psi\left[v^{0}, v^{+}\right]:=v^{1} .
$$

Then we have

$$
u^{-} \leq \Psi\left[u^{0}, u^{-}\right] \leq \Psi\left[v^{0}, v^{+}\right] \leq v^{+}
$$

and

$$
\Psi\left[u^{0}, u\right]=u \quad \text { if and only if } \quad u \text { is a solution . }
$$

Proof.

By applying Proposition 6.7 repeatedly, we get the following chain of inequalities:

$$
u^{-} \leq \Phi\left[u^{0}, u^{-, k}\right] \leq \Phi\left[u^{0}, u^{-, k+1}\right] \leq \ldots \leq \Phi\left[v^{0}, v^{+, k+1}\right] \leq \Phi\left[v^{0}, v^{+, k}\right] \leq v^{+} .
$$

Then the sequence $u^{-, k}$ is non-decreasing and bounded from above by $v^{+}$, whereas $v^{+, k}$ is non-increasing and bounded from below by $u^{-}$. Taking the limit as $k$ goes to infinity we conclude

$$
u^{-} \leq \lim _{k \rightarrow \infty} u^{-, k}=: u^{1}=: \Psi\left[u^{0}, u^{-}\right] \leq \Psi\left[v^{0}, v^{+}\right]:=v^{1}:=\lim _{k \rightarrow \infty} v^{+, k} \leq v^{+} .
$$

Now we prove that $u^{1}$ and $v^{1}$ are solutions of the scheme at step $\Delta t$, respectively with initial data $u^{0}$ and $v^{0}$. By definition of the map $\Phi$, the sequence $u^{-, k}$ satisfies, for all $k \in \mathbb{N}$ and $I \in \mathbb{Z}^{N}$,

$$
u_{I}^{0}+\Delta t\left(R_{*}\right)_{I}\left[u^{-, k}\right]\left(u^{-, k+1}\right) \leq u^{-, k+1} \leq u_{I}^{0}+\Delta t R_{I}^{*}\left[u^{-, k}\right]\left(u^{-, k+1}\right) .
$$

Moreover, by continuity of discrete gradients and semi-continuity of $E^{*}, E_{*}$, we easily obtain that $R^{*}, R_{*}$ are respectively upper and lower semicontinuous. Then by taking the limit as $k \rightarrow+\infty$ in (6.5), we get

$$
u_{I}^{0}+\Delta t\left(R_{*}\right)_{I}\left[u^{1}\right] \leq u^{1} \leq u_{I}^{0}+\Delta t R_{I}^{*}\left[u^{1}\right],
$$

i.e., $u^{1}$ is a solution. Similarly we conclude that $v^{1}$ is also a solution. Finally, the property $\Psi\left[u^{0}, u\right]=u$ if and only if $u$ is a solution directly follows by the corresponding property of the map $\Phi$ (see Proposition 6.5) .

As stated in the next result, the solutions constructed above are extremal solutions in the interval $\left[u^{-}, v^{+}\right]$, i.e., $\Psi\left[u^{0}, u^{-}\right]$is the minimal solution above $u^{-}$and $\Psi\left[v^{0}, v^{+}\right]$is the maximal solution below $v^{+}$, among all the solutions $w$ with initial datum $w^{0}$ such that $u^{-} \leq w \leq v^{+}$and $u^{0} \leq w^{0} \leq v^{0}$.

Proposition 6.9 (Extremal solutions in the window $\left[u^{-}, v^{+}\right]$)

Let $\Psi\left[u^{0}, u^{-}\right], \Psi\left[v^{0}, v^{+}\right]$be the solutions of the scheme (3.4) given by Proposition 6.8 and consider a discrete function $w^{0}$ such that $u^{0} \leq w^{0} \leq v^{0}$. Then every solution $w$ at step $\Delta t$ with initial datum $w^{0}$ such that $u^{-} \leq w \leq v^{+}$satisfies

$$
\Psi\left[u^{0}, u^{-}\right] \leq w \leq \Psi\left[v^{0}, v^{+}\right] .
$$

Proof.

Every solution $w$ above $u^{-}$is in particular a super-solution. Then, by applying Proposition 6.8 to $u^{-}$and $w$, we get

$$
u^{-} \leq \Psi\left[u^{0}, u^{-}\right] \leq \Psi\left[w^{0}, w\right]=w .
$$

The proof of the maximality of $\Psi\left[v^{0}, v^{+}\right]$is similar and we omit it.

Remark 6.10 We remark here an important point: if we choose the barriers given by Proposition 4.2 as the initial sub and super solutions $u^{-}, v^{+}$of our construction, we recover exactly the extremal solutions obtained in Theorem 6.3 by the Perron's method (see Remark 6.4). But now the situation is somehow reversed, the minimal solution is reached from below by sub-solutions, whereas the maximal one is reached from above by super-solutions. 
Now we are in a position to prove Theorem 1.5. The idea is just to apply the previous construction iteratively in time.

\section{Proof of Theorem 1.5.}

Step (i): construction of extremal solutions

We first consider the discrete barriers given by Proposition 4.2, namely

$$
v_{I}^{ \pm, n}=v_{I}^{0} \pm C_{v^{0}} n \Delta t, \quad \text { for all } I \in \mathbb{Z}^{N}, n \in \mathbb{N}, \quad \text { with } \quad C_{v^{0}} \geq \max \left\{\left\|R_{*}\left[v^{0}\right]\right\|_{\infty},\left\|R^{*}\left[v^{0}\right]\right\|_{\infty}\right\}
$$

and we recall that every solution $v$ of the scheme (3.4) has to satisfy

$$
v_{I}^{-, n} \leq v_{I}^{n} \leq v_{I}^{+, n} \quad \text { for all } I \in \mathbb{Z}^{N}, n \in \mathbb{N} .
$$

Now we construct the extremal solutions $\underline{v}$ and $\bar{v}$ by recurrence in $n$.

For $n=0$ we set $\underline{v}^{0}=\bar{v}^{0}=v^{0}$ and we remark that, by definition, the functions $v^{-, 1}, v^{+, 1}$ are respectively a sub-solution and a super-solution of the scheme at step 1 with initial datum $v^{0}$. By Proposition 6.8 it follows that

$$
\underline{v}^{1}:=\Psi\left[v^{0}, v^{-, 1}\right] \quad \text { and } \quad \bar{v}^{1}:=\Psi\left[v^{0}, v^{+, 1}\right]
$$

are two solutions a step 1. Moreover by (6.6) and Proposition 6.9 we get

$$
v^{-, 1} \leq \underline{v}^{1} \leq v^{1} \leq \bar{v}^{1} \leq v^{+, 1} .
$$

Now let $n \geq 1$. We assume

$$
v^{-, n} \leq \underline{v}^{n} \leq v^{n} \leq \bar{v}^{n} \leq v^{+, n}
$$

and define

$$
\underline{v}^{n+1}:=\Psi\left[\underline{v}^{n}, v^{-, n+1}\right] \quad \text { and } \quad \bar{v}^{n+1}:=\Psi\left[\bar{v}^{n}, v^{+, n+1}\right] .
$$

We remark that this definition is well posed if $v^{-, n+1}$ is a sub-solution at step 1 with initial datum $\underline{v}^{n}$ and $v^{+, n+1}$ is a super-solution at step 1 with initial datum $\bar{v}^{n}$. Indeed, by (6.7) we get for all $I \in \mathbb{Z}^{N}$

$$
\frac{v_{I}^{-, n+1}-\underline{v}_{I}^{n}}{\Delta t}=\frac{v_{I}^{-, n+1}-v_{I}^{-, n}}{\Delta t}+\frac{v_{I}^{-, n}-\underline{v}_{I}^{n}}{\Delta t} \leq-C_{v^{0}} \leq R_{I}^{*}\left[v^{0}\right]=R_{I}^{*}\left[v^{-, n+1}\right]
$$

and

$$
\frac{v_{I}^{+, n+1}-\bar{v}_{I}^{n}}{\Delta t}=\frac{v_{I}^{+, n+1}-v_{I}^{+, n}}{\Delta t}+\frac{v_{I}^{+, n}-\bar{v}_{I}^{n}}{\Delta t} \geq C_{v^{0}} \geq\left(R_{*}\right)_{I}\left[v^{0}\right]=\left(R_{*}\right)_{I}\left[v^{+, n+1}\right] .
$$

By Proposition 6.9 we conclude that

$$
v^{-, n+1} \leq \underline{v}^{n+1} \leq v^{n+1} \leq \bar{v}^{n+1} \leq v^{+, n+1} .
$$

Step (ii): construction of a solution with monotone time derivatives For the time step $n=0$ we set

$$
\begin{gathered}
\underline{\lambda}^{0}=\inf _{I \in \mathbb{Z}^{N}} R_{I}^{*}\left[v^{0}\right], \quad \bar{\lambda}^{0}=\sup _{I \in \mathbb{Z}^{N}}\left(R_{*}\right)_{I}\left[v^{0}\right], \quad \delta=\max \left\{\underline{\lambda}^{0}-\bar{\lambda}^{0}, 0\right\}, \\
\underline{\mu}^{0}=\underline{\lambda}^{0}-\delta / 2, \quad \bar{\mu}^{0}=\bar{\lambda}^{0}+\delta / 2 .
\end{gathered}
$$

and we define for all $I \in \mathbb{Z}^{N}$

$$
v_{I}^{-}=v_{I}^{0}+\Delta t \underline{\mu}^{0}, \quad v_{I}^{+}=v_{I}^{0}+\Delta t \bar{\mu}^{0},
$$

so that we have $\mu^{0} \leq \bar{\mu}^{0}$ and $v^{-} \leq v^{+}$. Moreover $v^{-}, v^{+}$are respectively a sub-solution and a super-solution at step 1 with initial datum $v^{0}$. Then we denote by $v^{1}$ one of the two solutions $\Psi\left[v^{0}, v^{-}\right], \Psi\left[v^{0}, v^{+}\right]$given by Proposition 6.8, applied to $v^{-}$and $v^{+}$. It follows that $v^{-} \leq v^{1} \leq v^{+}$, which implies for all $I \in \mathbb{Z}^{N}$

$$
\underline{\mu}^{0}=\frac{v_{I}^{-}-v_{I}^{0}}{\Delta t} \leq \inf _{I \in \mathbb{Z}^{N}}\left(\frac{v_{I}^{1}-v_{I}^{0}}{\Delta t}\right)=: \underline{\mu}^{1} \leq \bar{\mu}^{1}:=\sup _{I \in \mathbb{Z}^{N}}\left(\frac{v_{I}^{1}-v_{I}^{0}}{\Delta t}\right) \leq \frac{v_{I}^{+}-v_{I}^{0}}{\Delta t}=\bar{\mu}^{0} .
$$


Similarly, for every $n \geq 1$, we set

$$
\underline{\mu}^{n}=\inf _{I \in \mathbb{Z}^{N}}\left(\frac{v_{I}^{n}-v_{I}^{n-1}}{\Delta t}\right), \quad \bar{\mu}^{n}=\sup _{I \in \mathbb{Z}^{N}}\left(\frac{v_{I}^{n}-v_{I}^{n-1}}{\Delta t}\right)
$$

and define for all $I \in \mathbb{Z}^{N}$

$$
v_{I}^{-}=v_{I}^{n}+\Delta t \underline{\mu}^{n}, \quad v_{I}^{+}=v_{I}^{n}+\Delta t \bar{\mu}^{n} .
$$

By construction we have $\mu^{n} \leq \bar{\mu}^{n}$ and $v^{-} \leq v^{+}$. Moreover $v^{-}, v^{+}$are respectively a sub-solution and a super-solution at step $n+\overline{1}$. Then we construct the solution $v^{n+1}$ as previously and we conclude that

$$
\underline{\mu}^{n} \leq \underline{\mu}^{n+1} \leq \bar{\mu}^{n+1} \leq \bar{\mu}^{n} .
$$

The proof is complete.

Remark 6.11 In order to obtain a better approximation of the effective Hamiltonian, we can apply Theorem 1.3 to the solution $v$ given by Theorem 1.5-(ii): for every $n \in \mathbb{N}$ we get

$$
\underline{\lambda}_{v^{n}}^{d}-C_{v^{n}} \sqrt{\Delta x} \leq \lambda \leq \bar{\lambda}_{v^{n}}^{d}+C_{v^{n}} \sqrt{\Delta x} .
$$

Since $v$ is both a sub-solution and a super-solution, we have for all $n \in \mathbb{N}$

$$
\begin{gathered}
\underline{\mu}^{n}=\inf _{I \in \mathbb{Z}^{N}}\left(\frac{v_{I}^{n}-v_{I}^{n-1}}{\Delta t}\right) \leq \inf _{I \in \mathbb{Z}^{N}} R_{I}^{*}\left[v^{n}\right]=\underline{\lambda}_{v^{n}}^{d}, \\
\bar{\mu}^{n}=\sup _{I \in \mathbb{Z}^{N}}\left(\frac{v_{I}^{n}-v_{I}^{n-1}}{\Delta t}\right) \geq \sup _{I \in \mathbb{Z}^{N}}\left(R_{*}\right)_{I}\left[v^{n}\right]=\bar{\lambda}_{v^{n}}^{d}
\end{gathered}
$$

and then

$$
\underline{\mu}^{n}-C_{v^{n}} \sqrt{\Delta x} \leq \lambda \leq \bar{\mu}^{n}+C_{v^{n}} \sqrt{\Delta x} .
$$

This estimate can improve if the constant $C_{v^{n}}$ remains controlled as $n$ goes to infinity, since the gap $\bar{\mu}^{n}-\mu^{n}$ is non-increasing in $n$ by Theorem 1.5. The same argument applies to the improved error estimate (5.1) in the one-dimensional case.

\section{$7 \quad$ Numerical Simulations}

In this section we present some numerical tests, concerning the approximation of the effective Hamiltonian. In order to highlight the features of the scheme (3.4), we mainly focus on the one-dimensional case. Nevertheless, we will also show simulations in the two-dimensional case at the end of the section.

\subsection{Setting of the computation}

First of all we have to reduce the non-local velocity

$$
\left(c^{*}\right)_{i}^{d}[v]=c_{0}\left(x_{i}\right)+\int_{\mathbb{R}} \mathcal{J}(z)\left\{E^{*}\left(v_{\sharp}\left(x_{i}+z\right)-v_{\sharp}\left(x_{i}\right)\right)-p z\right\} d z, \quad i \in \mathbb{Z}
$$

in a form suitable for numerical computations. To this end we set $\Delta x=1 / N_{0}$ with $N_{0} \in \mathbb{N} \backslash\{0\}, p=P / Q$ with $P \in \mathbb{Z}, Q \in \mathbb{N} \backslash\{0\}$ and we consider discrete functions of the form periodic plus linear, namely $v_{i}=w_{i}+p x_{i}$, where $w$ is $\frac{1}{\Delta x}$-periodic function. By taking the following partition of $\mathbb{R}$ in intervals of size 1 , i.e.

$$
\mathbb{R}=\bigcup_{h \in \mathbb{Z}} \bigcup_{m=0}^{Q-1}[h Q+m-\Delta x / 2, h Q+m+1-\Delta x / 2),
$$

and by a change of variables, we can rewrite the integral in $\left(c^{*}\right)_{i}^{d}[v]$ as

$$
I:=\sum_{h \in \mathbb{Z}} \sum_{m=0}^{Q-1} \int_{-\Delta x / 2}^{1-\Delta x / 2} \mathcal{J}(z+h Q+m)\left\{E^{*}\left(v_{\sharp}\left(x_{i}+z+h Q+m\right)-v_{\sharp}\left(x_{i}\right)\right)-p(z+h Q+m)\right\} d z .
$$


Since $[-\Delta x / 2,1-\Delta x / 2)=\bigcup_{j=0}^{N_{0}-1}\left[x_{j}-\Delta x / 2, x_{j}+\Delta x / 2\right)$ and the function $v_{\sharp}$ is constant on each interval, we obtain

$$
I=\sum_{h \in \mathbb{Z}} \sum_{m=0}^{Q-1} \sum_{j=0}^{N_{0}-1} \int_{x_{j}-\Delta x / 2}^{x_{j}+\Delta x / 2} \mathcal{J}(z+h Q+m)\left\{E^{*}\left(v_{\sharp}\left(x_{i}+x_{j}+h Q+m\right)-v_{\sharp}\left(x_{i}\right)\right)-p(z+h Q+m)\right\} d z .
$$

Now

$$
\begin{gathered}
v_{\sharp}\left(x_{i}+x_{j}+h Q+m\right)-v_{\sharp}\left(x_{i}\right)= \\
=w_{\sharp}\left(x_{i}+x_{j}+h Q+m\right)+p\left(x_{i}+x_{j}+h Q+m\right)-w_{\sharp}\left(x_{i}\right)-p x_{i}= \\
=w_{\sharp}\left(x_{i}+x_{j}\right)-w_{\sharp}\left(x_{i}\right)+p\left(x_{j}+h Q+m\right)= \\
=w_{i+j}-w_{i}+p\left(x_{j}+m\right)+p h Q,
\end{gathered}
$$

where we used the fact that $h Q+m \in \mathbb{Z}$ and $w_{\sharp}$ is a 1 -periodic function. Moreover $p h Q \in \mathbb{Z}$ and

$$
E^{*}\left(w_{i+j}-w_{i}+p\left(x_{j}+m\right)+p h Q\right)=E^{*}\left(w_{i+j}-w_{i}+p\left(x_{j}+m\right)\right)+p h Q .
$$

Then we obtain

$$
I=\sum_{m=0}^{Q-1} \sum_{j=0}^{N_{0}-1} \int_{x_{j}-\Delta x / 2}^{x_{j}+\Delta x / 2} \mathcal{J}^{Q}(z+m)\left\{E^{*}\left(w_{i+j}-w_{i}+p\left(x_{j}+m\right)\right)-p(z+m)\right\} d z
$$

where $\mathcal{J}^{Q}$ is the $Q$-periodic kernel given by

$$
\mathcal{J}^{Q}(t)=\sum_{h \in \mathbb{Z}} \mathcal{J}(t+h Q) .
$$

We now define

$$
\mathcal{J}_{m, j}^{Q}=\frac{1}{\Delta x} \int_{x_{j}-\Delta x / 2}^{x_{j}+\Delta x / 2} \mathcal{J}^{Q}(z+m) d z \quad \text { and } \quad \zeta_{m, j}^{Q}=\frac{1}{\Delta x} \int_{x_{j}-\Delta x / 2}^{x_{j}+\Delta x / 2}(z+m) \mathcal{J}^{Q}(z+m) d z .
$$

It follows that

$$
I=\sum_{m=0}^{Q-1} \sum_{j=0}^{N_{0}-1}\left\{\mathcal{J}_{m, j}^{Q} E^{*}\left(w_{i+j}-w_{i}+p\left(x_{j}+m\right)\right)-p \zeta_{m, j}^{Q}\right\} \Delta x
$$

We remark that the coefficients $\mathcal{J}_{m, j}^{Q}$ and $\zeta_{m, j}^{Q}$ can be pre-computed for a given $Q$ and $\Delta x$. In practice we use a simple rectangular quadrature rule in order to approximate the integrals in (7.3) and a large (but finite) sum to sample the kernel $\mathcal{J}^{Q}$ in (7.2): for some $N_{1} \in \mathbb{N}$

$$
\mathcal{J}^{Q}(t) \simeq \sum_{h=-N_{1}}^{N_{1}} \mathcal{J}(t+h Q), \quad \mathcal{J}_{m, j}^{Q} \simeq \mathcal{J}^{Q}\left(x_{j}+m\right) \quad \text { and } \quad \zeta_{m, j}^{Q} \simeq\left(x_{j}+m\right) \mathcal{J}^{Q}\left(x_{j}+m\right)
$$

With this choice we need $N_{0} Q$ pre-computations and the non-local velocity $\left(c^{*}\right)_{i}^{d}[v]$, for $i=0, \ldots, N_{0}-1$, reduces to

$$
\left(c^{*}\right)_{i}^{d}[v] \simeq c_{0}\left(x_{i}\right)+\sum_{m=0}^{Q-1} \sum_{j=0}^{N-1} \mathcal{J}_{m, j}^{Q}\left\{E^{*}\left(w_{i+j}-w_{i}+p\left(x_{j}+m\right)\right)-p\left(x_{j}+m\right)\right\} \Delta x=:\left(c^{*}\right)_{i}^{b}[w] .
$$

Similarly, by replacing $E^{*}$ with $E_{*}$, we obtain the following approximation of $\left(c_{*}\right)_{i}^{d}[v]$ :

$$
\left(c_{*}\right)_{i}^{d}[v] \simeq c_{0}\left(x_{i}\right)+\sum_{m=0}^{Q-1} \sum_{j=0}^{N-1} \mathcal{J}_{m, j}^{Q}\left\{E_{*}\left(w_{i+j}-w_{i}+p\left(x_{j}+m\right)\right)-p\left(x_{j}+m\right)\right\} \Delta x=:\left(c_{*}\right)_{i}^{b}[w] .
$$

Then we define

$$
\left(R^{*}\right)_{i}^{b}[w]=\left(c^{*}\right)_{i}^{b}[w] G^{\left(s^{*}\right)_{i}[w]}\left(D w_{i}+p\right) \quad \text { with }\left(s^{*}\right)_{i}[w]=\operatorname{sign}\left(\left(c^{*}\right)_{i}^{b}[w]\right),
$$




$$
\left(R_{*}\right)_{i}^{b}[w]=\left(c_{*}\right)_{i}^{b}[w] G^{\left(s_{*}\right)_{i}[w]}\left(D w_{i}+p\right) \quad \text { with }\left(s_{*}\right)_{i}[w]=\operatorname{sign}\left(\left(c_{*}\right)_{i}^{b}[w]\right)
$$

and we compute the discrete effective Hamiltonian by following the time evolution of approximate sub and super solutions of the implicit scheme (3.4). In terms of $w$ we have to solve

$$
w_{i}^{0}=0, \quad \frac{w_{i}^{n+1}-w_{i}^{n}}{\Delta t}=(R)_{i}^{b}\left[w^{n+1}\right] \quad i=0, \ldots, N_{0}-1, n \in \mathbb{N},
$$

in the sense of discrete viscosity solutions given in Definition 3.4.

\subsection{The algorithm}

We introduce three parameters $\varepsilon_{d}, \varepsilon_{c}, \varepsilon_{s}>0$, respectively a tolerance to quit the dichotomy process updating sub and super solutions, a tolerance for the convergence of the scheme for a single time step $\Delta t$ and a tolerance for the saturation in time of the values approximating the effective Hamiltonian. Then we implement, for a given $p \in \mathbb{R}$, the following algorithm:

1) Initialization for $n=0$ :

$$
\begin{aligned}
& w_{i}^{-, 0}=w_{i}^{+, 0}=w_{i}^{0}=0, \quad \underline{\lambda}^{0}=\min _{i=0, \ldots, N_{0}-1}\left(R^{*}\right)_{i}^{b}\left[w^{0}\right], \quad \bar{\lambda}^{0}=\max _{i=0, \ldots, N_{0}-1}\left(R_{*}\right)_{i}^{b}\left[w^{0}\right], \\
& \delta=\max \left\{\underline{\lambda}^{0}-\bar{\lambda}^{0}, 0\right\}, \quad \underline{\mu}^{0}=\underline{\lambda}^{0}-\delta / 2, \quad \bar{\mu}^{0}=\bar{\lambda}^{0}+\delta / 2 .
\end{aligned}
$$

2) Build initial sub-solution and super-solution for current step $n$ :

$$
w_{i}^{-}=w_{i}^{-, n}+\Delta t \underline{\mu}^{n} \quad \text { and } \quad w_{i}^{+}=w_{i}^{+, n}+\Delta t \bar{\mu}^{n}, \quad i=0, \ldots, N_{0}-1 .
$$

3) Initialize dichotomy intervals: for $i=0, \ldots, N_{0}-1$ set

$$
s_{\text {left }, i}^{-}=w_{i}^{-}, \quad s_{\text {right }, i}^{-}=w_{i}^{-}+k_{i}^{-},
$$

with $k_{i}^{-} \in \mathbb{N}$ the first integer such that

$$
s_{\text {right }, i}^{-}>w_{i}^{-, n}+\Delta t\left(R^{*}\right)_{i}^{b}\left[w^{-}\right]\left(s_{\text {right }, i}^{-}\right)
$$

and

$$
s_{\text {left }, i}^{+}=w_{i}^{+}-k_{i}^{+}, \quad s_{\text {right }, i}^{+}=w_{i}^{+},
$$

with $k_{i}^{+} \in \mathbb{N}$ the first integer such that

$$
s_{\text {left }, i}^{+}<w_{i}^{-, n}+\Delta t\left(R_{*}\right)_{i}^{b}\left[w^{-}\right]\left(s_{\text {left }, i}^{+}\right) .
$$

4) Dichotomy process: for $i=0, \ldots, N_{0}-1$ freeze all the values $w_{j}^{-}, w_{j}^{+}$with $j \neq i$ and optimize, respectively in $s_{i}^{-} \in\left[s_{\text {left }, i}^{-}, s_{\text {right }, i}^{-}\right)$and $s_{i}^{+} \in\left[s_{\text {left }, i}^{+}, s_{\text {right }, i}^{+}\right)$, the inequalities

$$
s_{i}^{-} \leq w_{i}^{-, n}+\Delta t\left(R^{*}\right)_{i}^{b}\left[w^{-}\right]\left(s_{i}^{-}\right) \quad \text { and } \quad s_{i}^{+} \geq w_{i}^{+, n}+\Delta t\left(R_{*}\right)_{i}^{b}\left[w^{+}\right]\left(s_{i}^{+}\right)
$$

until $s_{\text {right }, i}^{-}-s_{\text {left }, i}^{-}<\varepsilon_{d}$ and $s_{\text {right }, i}^{+}-s_{\text {left }, i}^{+}<\varepsilon_{d}$.

$5)$ Build auxiliary sub-solution and super-solution: for $i=0, \ldots, N_{0}-1$ set

$$
\left(w_{\text {aux }}^{-}\right)_{i}=s_{\text {left }, i}^{-} \quad \text { and } \quad\left(w_{\text {aux }}^{+}\right)_{i}=s_{\text {right }, i}^{+} .
$$

If $\left\|w^{-}-w_{\text {aux }}^{-}\right\|_{\infty}<\varepsilon_{c}$ and $\left\|w^{+}-w_{\text {aux }}^{+}\right\|_{\infty}<\varepsilon_{c}$ then go to step (6); else swap $w^{-} \leftrightarrow w_{\text {aux }}^{-}, w^{+} \leftrightarrow w_{\text {aux }}^{+}$ and go to step (3). 
6) Step forward in time: set

$$
w^{-, n+1}=w_{\text {aux }}^{-}, \quad \underline{\mu}^{n+1}=\min _{i=0, \ldots, N_{0}-1}\left(\frac{w_{i}^{-, n+1}-w_{i}^{-, n}}{\Delta t}\right)
$$

and

$$
w^{+, n+1}=w_{\mathrm{aux}}^{+}, \quad \bar{\mu}^{n+1}=\max _{i=0, \ldots, N_{0}-1}\left(\frac{w_{i}^{+, n+1}-w_{i}^{+, n}}{\Delta t}\right) .
$$

If $\left|\underline{\mu}^{n+1}-\underline{\mu}^{n}\right|<\varepsilon_{s}$ and $\left|\bar{\mu}^{n+1}-\bar{\mu}^{n}\right|<\varepsilon_{s}$ go to step (7); else go to step (2) with $n \leftrightarrow n+1$.

7) Set $n_{\text {last }}=n+1$ and exit: the value $\lambda$ of the effective Hamiltonian is approximated by the interval $\left[\underline{\mu}^{n_{\text {last }}}, \bar{\mu}^{n_{\text {last }}}\right]$.

Remark 7.1 By construction, we have

$$
\underline{\mu}^{n} \leq \underline{\mu}^{n+1} \quad \text { and } \quad \bar{\mu}^{n+1} \leq \bar{\mu}^{n} \quad \text { for all } n \in \mathbb{N} .
$$

Remark 7.2 In order to speed up the dichotomy process (step (4) of the algorithm), we can update $w_{i}^{-}$and $w_{i}^{+}$sequentially, as soon as the values $s_{i}^{-}$and $s_{i}^{+}$are computed.

\subsection{Tests in $1 \mathrm{D}$}

We consider a velocity of the form $c_{0}(x)=c_{1}(x)+L$, where $c_{1}: \mathbb{R} \rightarrow \mathbb{R}$ is a 1-periodic function representing obstacles to the motion of dislocations and $L \in \mathbb{R}$ is a driving force. We choose $N_{0}=100$ (i.e. $\Delta x=0.01$ ) and $Q=10$. Moreover $\Delta t=0.1, \varepsilon_{d}=10^{-9}, \varepsilon_{c}=10^{-6}$ and $\varepsilon_{s}=10^{-4}$. Finally we take $c_{1}(x)=A \sin (2 \pi x)$ with $A=2$ and the kernel $\mathcal{J}(z)=C_{\mathcal{J}} / z^{2}$ with $C_{\mathcal{J}}=1 / 2$, truncated inside the $\delta$-neighborhood of the origin of size $\delta=\Delta x$.

\subsubsection{Computation of the effective Hamiltonian}

Here we compute the effective Hamiltonian for $p, L \in[0,4]$ with steps $\Delta p=0.1$ and $\Delta L=0.1$. In Figure 1a we show an approximation of the effective Hamiltonian as a surface, obtained by taking for fixed $p$ and $L$ the minimal value $\mu^{n_{\text {last }}}$, while Figure $1 \mathrm{~b}$ represents the corresponding level-sets. For similar computations of
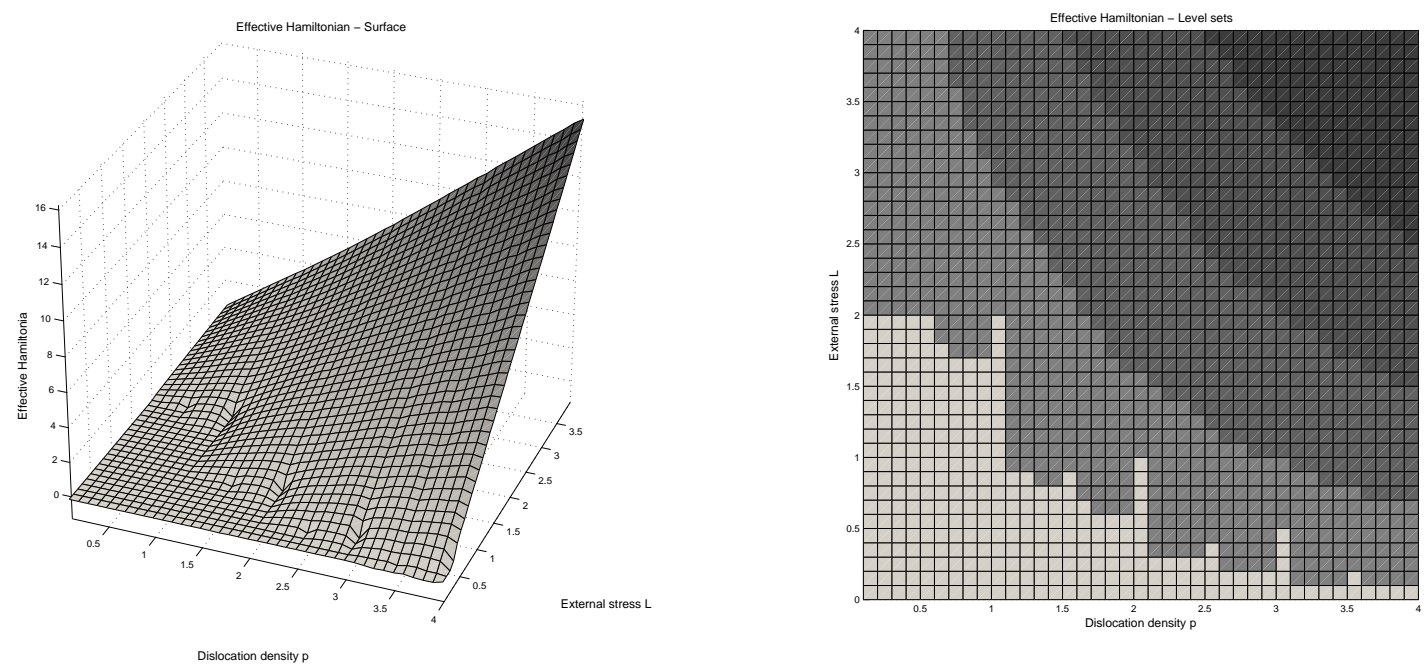

Figure 1: Surface graph (a) and level-sets (b) of the effective Hamiltonian. 
effective Hamiltonians with different methods or models, see [13],[16]. We remark that the zero level-set of the effective Hamiltonian (see the brightest grey region in Figure 1b) is very important, since it corresponds to the pinning of dislocations, namely the situation in which the external stress $L$ is small and dislocations can not overcome the obstacles given by the wells of $c_{1}$. On the other hand, when the density of dislocations $p$ increases, the non-local part of the velocity becomes relevant: dislocations exibit a cooperative behavior and are able to move inside the crystal lattice even if $L$ is not big enough. This situation is well illustrated by Figure 2, which shows the Hamiltonian as a function of $L$ for different values of $p$.

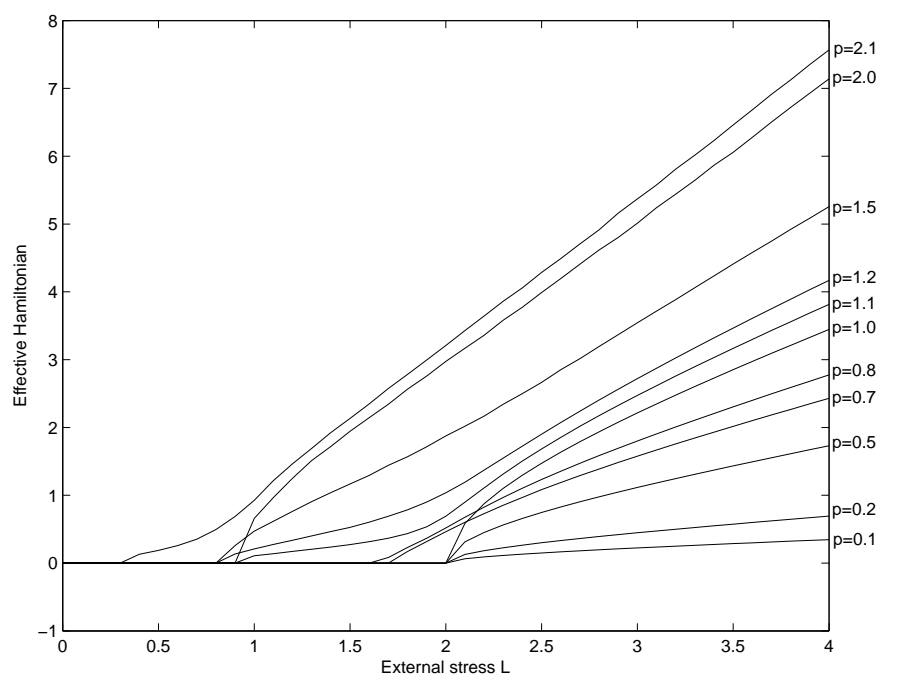

Figure 2: Graphs of the effective Hamiltonian as a function of $L$ for different values of $p$.

For small values of $p$ we have few dislocations in the interval $[0, Q]$, which are quite far from each other and then interact only weakly; we see in the simulation that the critical value $L=A=2$ is just the amount of the external stress needed to balance the size $A$ of the obstacles, a threshold between equilibrium and dynamics. While, for larger $p$, this threshold is drastically reduced, due to the fact that dislocations interact more and more.

\subsubsection{Monotonicity of time derivatives}

Here we show numerically the monotonicity property enjoyed by the time derivatives of discrete sub/super solutions $w^{-}, w^{+}$(see Remark 7.1) and we compare our approach of computing the effective Hamiltonian to the classical one. More precisely, for $1 \leq n \leq n_{\text {last }}$, we compare $\mu^{n}$ and $\bar{\mu}^{n}$ with

$$
\underline{\eta}^{n}=\min _{i=0, \ldots, N_{0}-1}\left(\frac{w_{i}^{-, n}}{n \Delta t}\right) \quad \text { and } \quad \bar{\eta}^{n}=\max _{i=0, \ldots, N_{0}-1}\left(\frac{w_{i}^{+, n}}{n \Delta t}\right) .
$$

In Figure 3 we show $\mu^{n}, \bar{\mu}^{n}$ and $\eta^{n}, \bar{\eta}^{n}$ as functions of $n$ in the case $p=0.5, L=2.3$. Despite the fact that the implicit scheme is quite expensive to solve, we clearly see that $\underline{\mu}^{n}, \bar{\mu}^{n}$ converge much faster than $\underline{\eta}^{n}, \bar{\eta}^{n}$.

\subsubsection{A posteriori error estimate}

This simulation concerns the a posteriori error estimate for the effective Hamiltonian $\lambda$. We consider the case $p=0.1, L=3$ and we compute $\mu^{n_{\text {last }}}, \bar{\mu}^{n_{\text {last }}}$ for different values of $\Delta x$, ranging from 0.1 to 0.01 . At each stage we restart the time evolution with initial data equal to the last $w^{ \pm, n_{\text {last }}}$, linearly interpolated on the new mesh. Then we check that the new sub/super solutions $v_{i}^{ \pm}:=w_{i}^{ \pm, n_{\text {last }}}+p x_{i}$ satisfy the assumptions of the improved error estimate given by Theorem 5.1 and we compute the corresponding constants $K_{v^{ \pm}}$in (5.2) such that

$$
-K_{v^{-}} \Delta x+\underline{\mu}^{n_{\text {last }}} \leq \lambda \leq \bar{\mu}^{n_{\text {last }}}+K_{v^{+}} \Delta x
$$




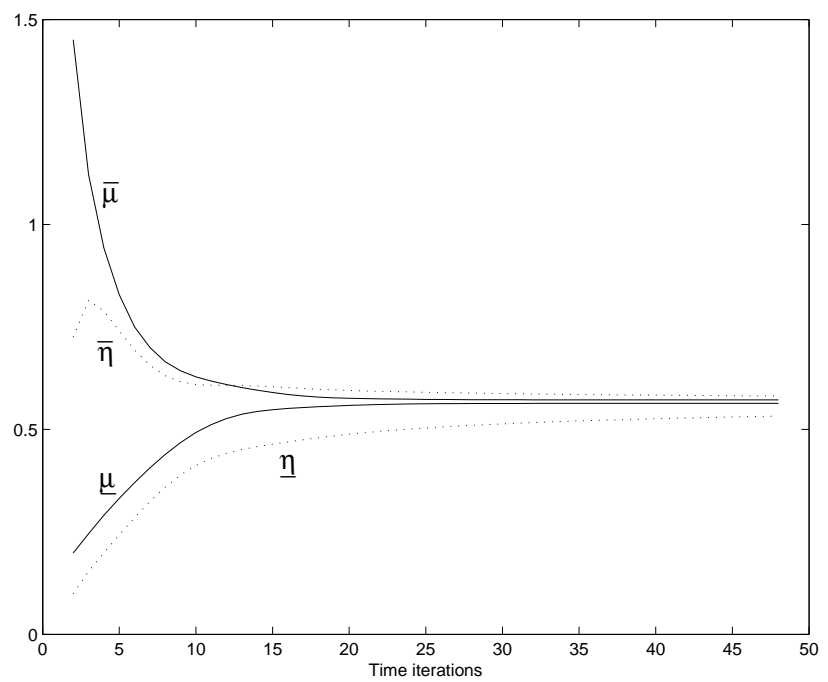

Figure 3: Monotonicity of time derivatives vs classical long time behavior.

Figure 4 shows $\mu^{n_{\text {last }}}, \bar{\mu}^{n_{\text {last }}}$ as functions of $\Delta x$ and also the inequalities (7.4) represented by error bars.

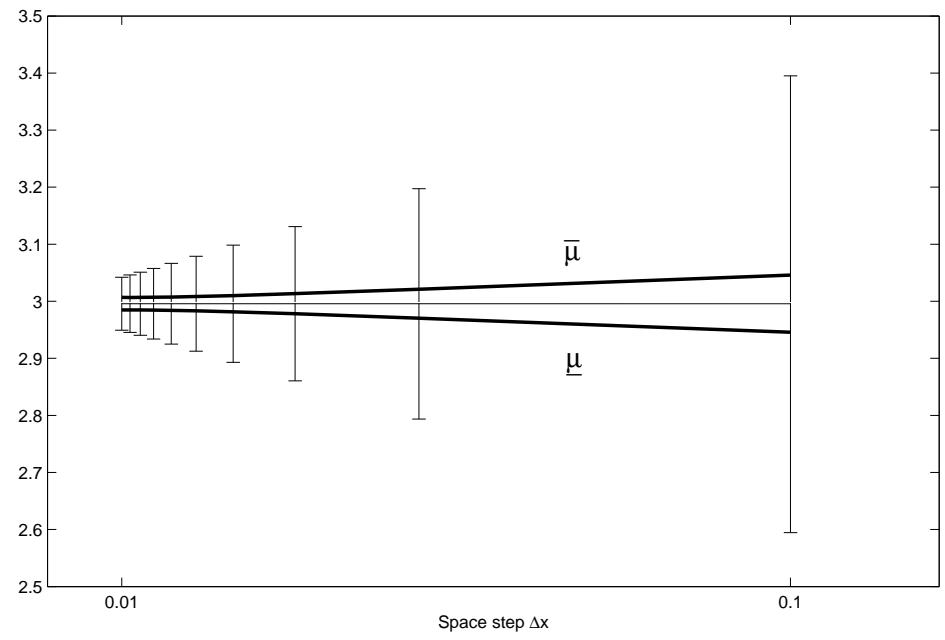

Figure 4: A posteriori error estimate of order $\Delta x$

\subsection{Tests in $2 \mathrm{D}$}

Here we present some simulations in the two-dimensional case. We recall that in our model what is physically meaningful is not the whole solution $v=w+p \cdot x$, but only its integer level-sets, representing dislocation lines in motion along a slip plane of the crystal. We then adopt this suggestive representation in all the figures below and show the time evolution through several key-frames. Moreover, in order to better appreciate the contribution of the non-local interactions between dislocations to the dynamics, we compare each simulation to its local counterpart. We just mention [9] for an alternative numerical method to compute the dynamics of a single dislocation line. We choose $N_{0}=20$ (i.e. $\Delta x=0.05$ ), $Q=1$ and $\Delta t=0.01$. Moreover we consider the periodic cell given by the square $[-Q / 2, Q / 2]^{2}$ and a velocity of the form $c_{0}(x)=c_{1}(x)+L$, 
with $c_{1}(x)=\min \left\{80|x|^{2}-1,0\right\}$, so that the obstacle to the motion of dislocations is just a circle. Finally, we take the kernel $\mathcal{J}(z)=C_{\mathcal{J}} /|z|^{3}$ with $C_{\mathcal{J}}=0.01$ and truncated inside the $\delta$-neighborhood of the origin of size $\delta=\Delta x$.

\subsubsection{Test 1: change of topology and self-interaction}

Here we consider a single dislocation line $(p=1)$ moving with a driving force $(L=1.1)$ greater than the size of the obstacle. Let us first examine the case without non-local interactions, namely the case $\mathcal{J} \equiv 0$.

As expected, Figure 5 shows that the line overcomes the obstacle, but it is partially trapped, so that it breaks changing its topology. Note that, inside the obstacle, the residual part shrinks and disappears in finite time, while the line keeps moving but remains distorted.

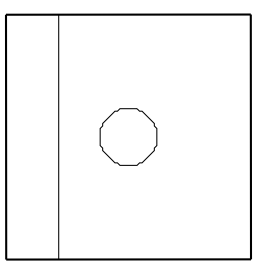

$t=0$

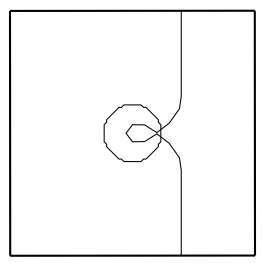

$t=0.44$

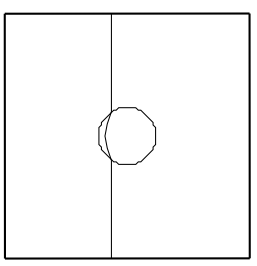

$t=0.2$

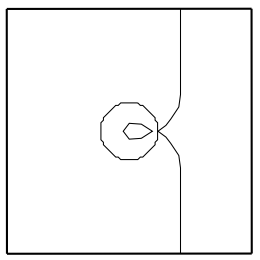

$t=0.45$

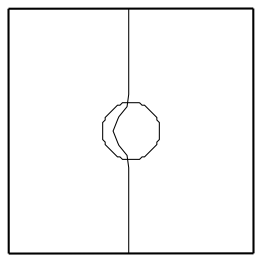

$t=0.25$

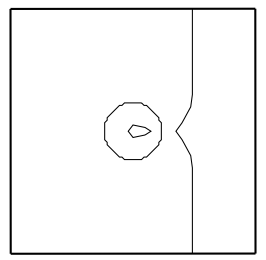

$t=0.48$

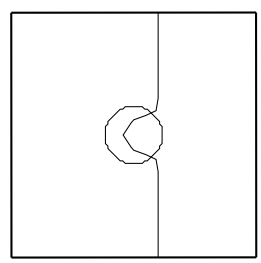

$t=0.35$

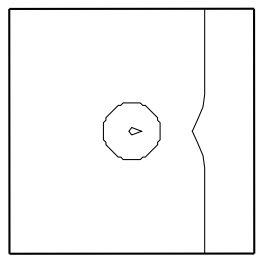

$t=0.53$

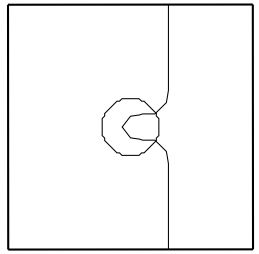

$t=0.4$

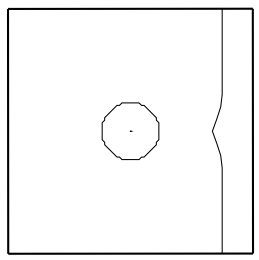

$t=0.6$

Figure 5: Evolution of a line without non-local interactions - change of topology

Now consider the non-local case with the same parameters. It is known that for a straight dislocation line there is no internal force created by self-interactions or, in other words, the non-local term in the velocity (7.1) is just zero if the periodic part of the solution $v=w+p \cdot x$ is constant. It follows that the line moves uniformly until it reaches the obstacle (see Figure 6). Then it starts bending and interacting with itself, so that it can exit the obstacle (without breaking in this simulation). Finally, it relaxes and becomes straight again.

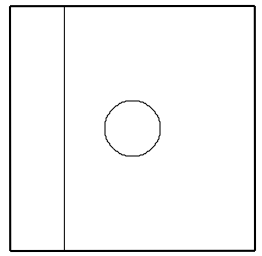

$t=0$

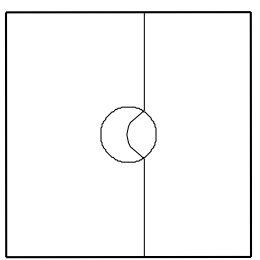

$t=0.4$

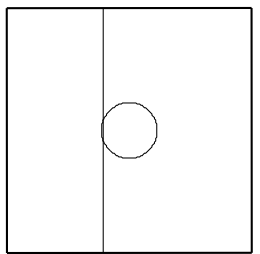

$t=0.2$

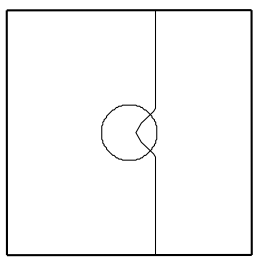

$t=0.45$

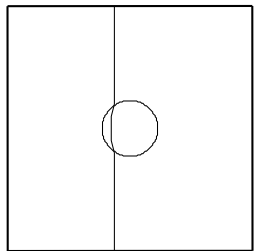

$\mathrm{t}=0.25$

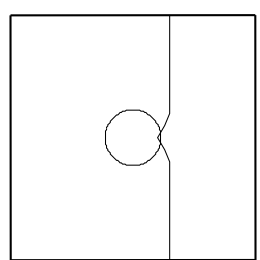

$t=0.5$

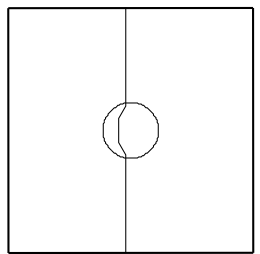

$\mathrm{t}=0.3$

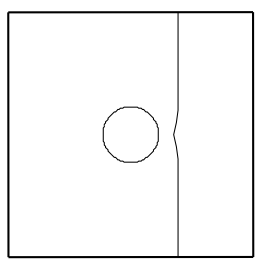

$t=0.55$

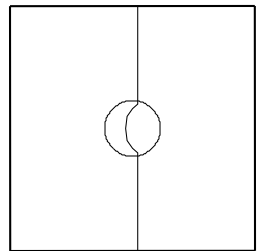

$\mathrm{t}=0.35$

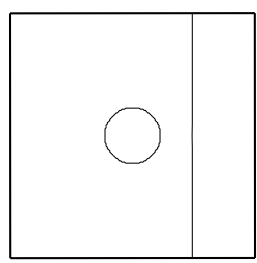

$t=0.6$

Figure 6: Evolution of a dislocation line with non-local self-interactions 


\subsubsection{Test 2: pile up effect}

We repeat Test 1 for the critical value $L=1$, i.e. we choose a driving force exactly equal to the size of the obstacle. In the case without interactions (Figure 7), we see again that the part of the line entering the obstacle has no hope to exit. Moreover we observe that every time the line passes through the obstacle (recall that we are in the periodic setting), new residuals pile up. Theoretically we expect that the loops will disappear in infinite time.

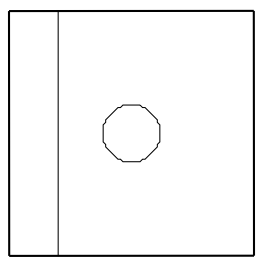

$t=0$

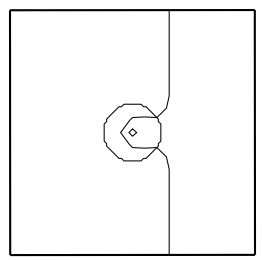

$\mathrm{t}=1.45$

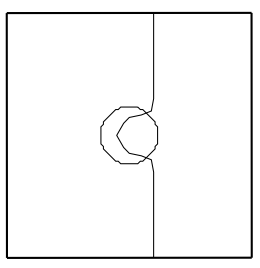

$\mathrm{t}=0.4$

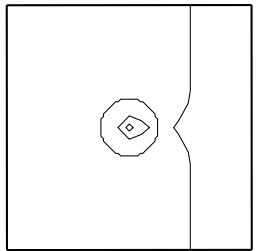

$t=1.55$

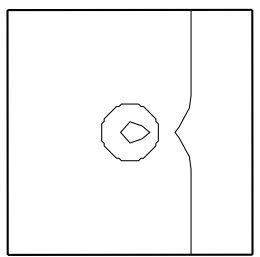

$\mathrm{t}=0.55$

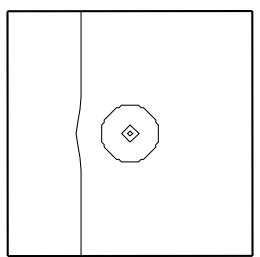

$\mathrm{t}=2.10$

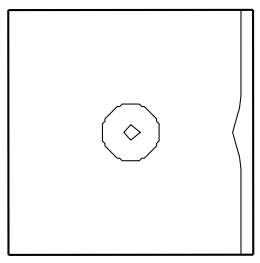

$\mathrm{t}=0.75$

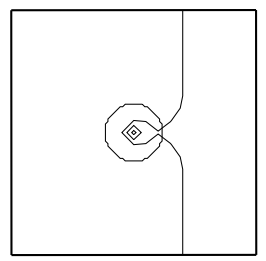

$\mathrm{t}=2.5$

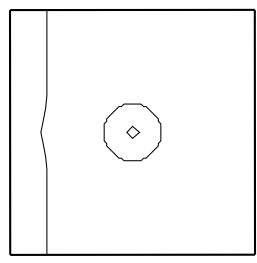

$\mathrm{t}=0.95$

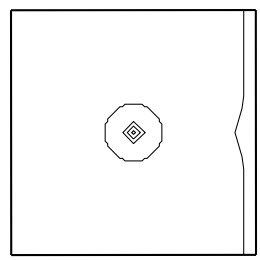

$\mathrm{t}=2.75$

Figure 7: Evolution of a line without non-local interactions - pile up effect

On the other hand, Figure 8 shows the computation in the non-local case. We see that the dislocation line can proceed "almost undisturbed", again thanks to self-interactions.

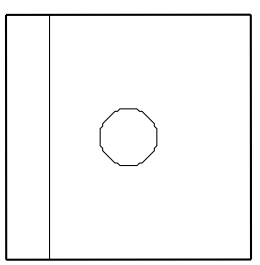

$t=0$

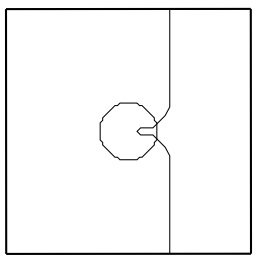

$\mathrm{t}=0.97$

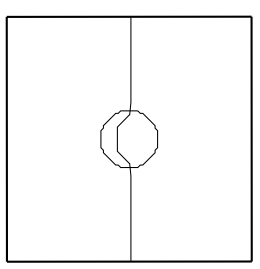

$t=0.65$

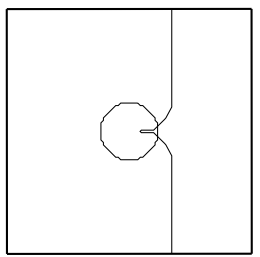

$t=0.98$

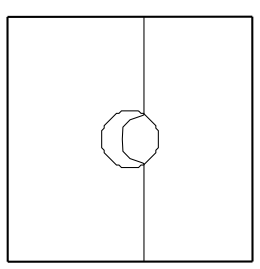

$t=0.75$

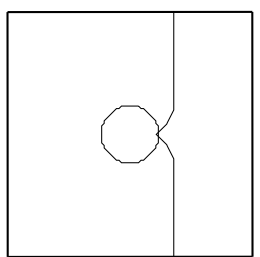

$t=0.99$

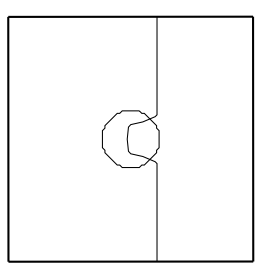

$t=0.85$

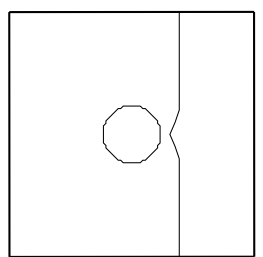

$t=1.02$

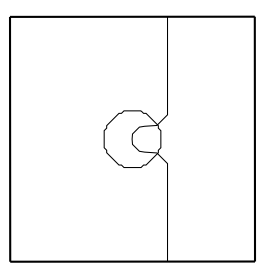

$t=0.92$

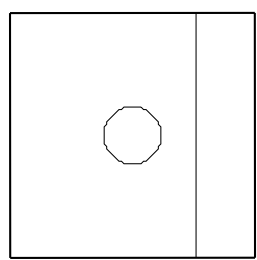

$t=1.15$

Figure 8: Evolution of a dislocation line with non-local self-interactions - "just a hitch" 


\subsubsection{Test 3: capturing of dislocations}

Here we consider again the motion of a single dislocation line $(p=1)$, but we change the velocity $c_{0}$ representing obstacles. More precisely, we choose the following discontinuous function:

$$
c_{0}(x)= \begin{cases}-0.5 & \text { if }|x|^{2} \leq 1 / 80 \\ 2 & \text { otherwise }\end{cases}
$$

Figure 9 corresponds to the case without interactions and we see a relevant difference with respect to the previous tests: the line does not enter the obstacle, but surroundes it. Then the line breaks and the residual loop is captured by the obstacle. This happens every time a new line comes, so we finally see a lot of loops piling up on the boundary of the circle.

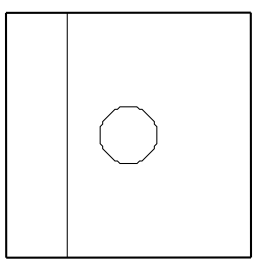

$\mathrm{t}=0$

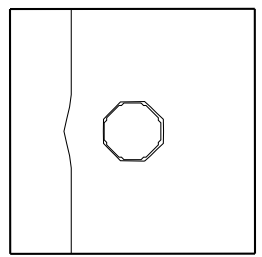

$\mathrm{t}=0.5$

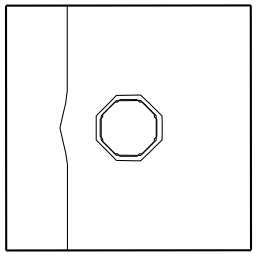

$\mathrm{t}=1$

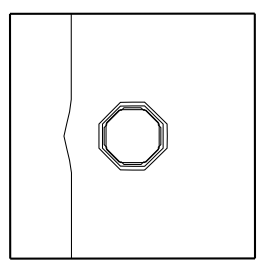

$t=1.5$

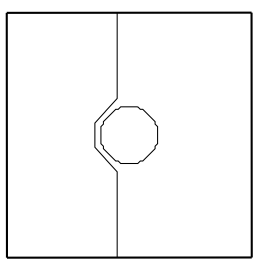

$t=0.1$

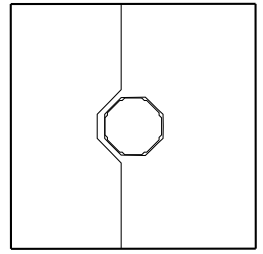

$t=0.6$

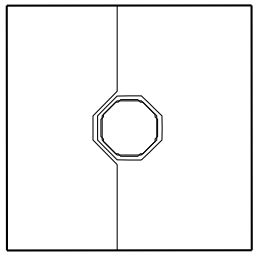

$t=1.1$

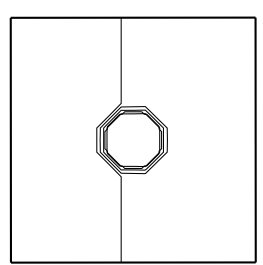

$t=1.6$

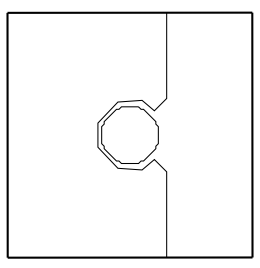

$\mathrm{t}=0.2$

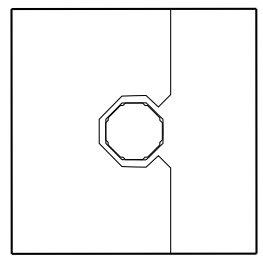

$\mathrm{t}=0.7$

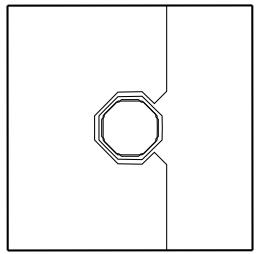

$\mathrm{t}=1.2$

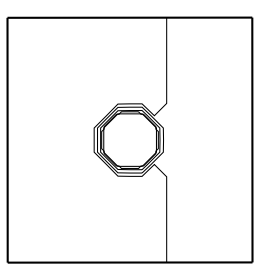

$t=1.7$

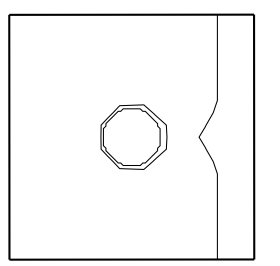

$t=0.3$

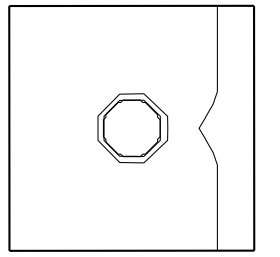

$\mathrm{t}=0.8$

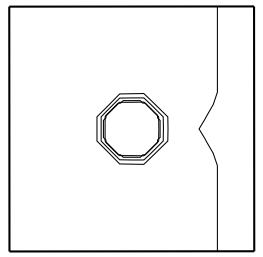

$t=1.3$

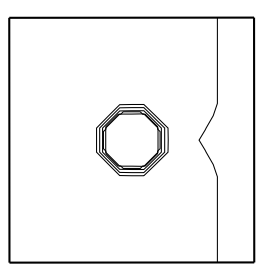

$t=1.8$

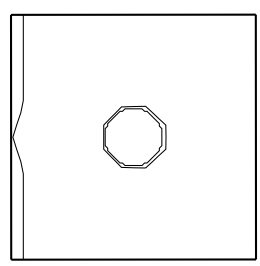

$t=0.4$

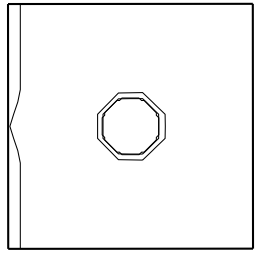

$\mathrm{t}=0.9$

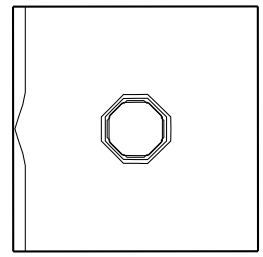

$\mathrm{t}=1.4$

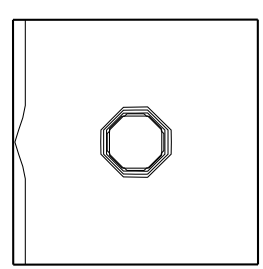

$t=1.9$

Figure 9: Capturing and piling up of loops without non-local interactions

The version with non-local interactions of this simulation is even more interesting and it is shown in Figure 10. Again, the dislocation line first surrounds the obstacle, then breaks and the residual part is quickly captured. But now a new line comes: it starts both surrounding the obstacle and pushing the dislocation loop. Since the force is not enough to climb the well, the residual dislocation shrinks and disappears, just while a new loop is created and captured. 


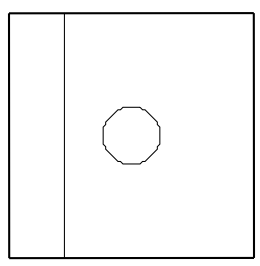

$t=0$

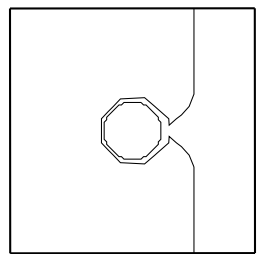

$t=0.3$

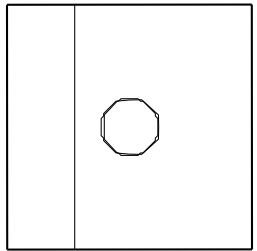

$t=0.6$

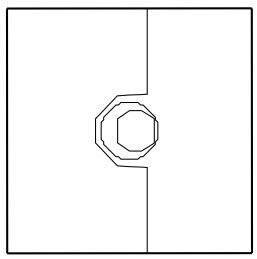

$t=0.77$

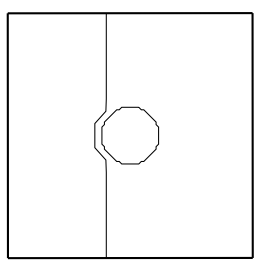

$\mathrm{t}=0.1$

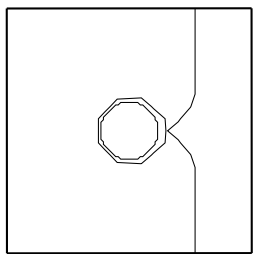

$t=0.31$

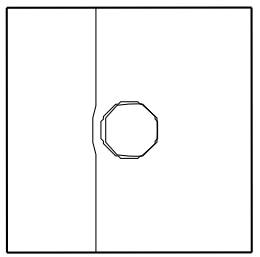

$t=0.65$

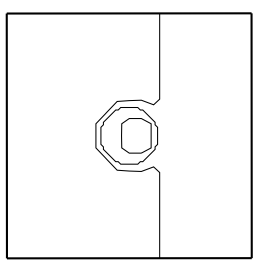

$\mathrm{t}=0.8$

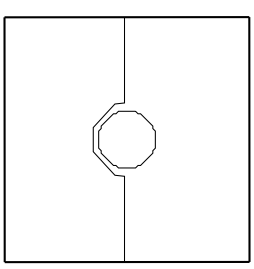

$t=0.15$

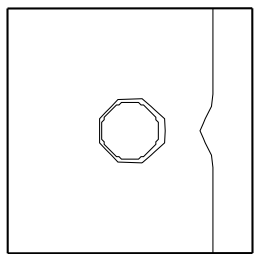

$\mathrm{t}=0.35$

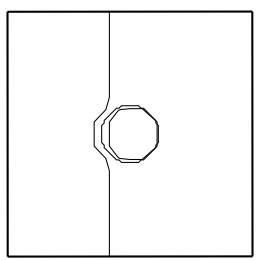

$t=0.68$

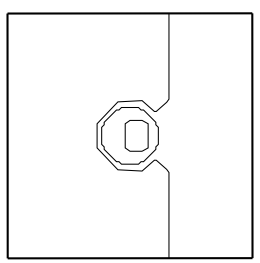

$t=0.82$

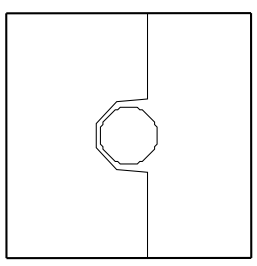

$t=0.2$

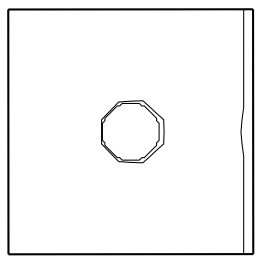

$t=0.42$

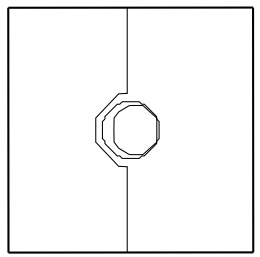

$t=0.72$

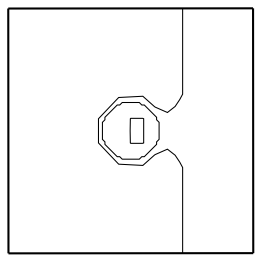

$t=0.85$

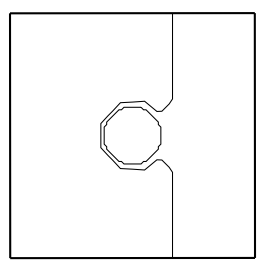

$t=0.25$

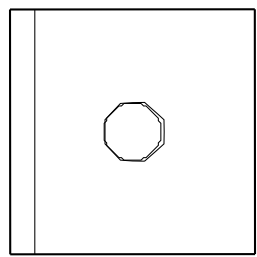

$t=0.5$

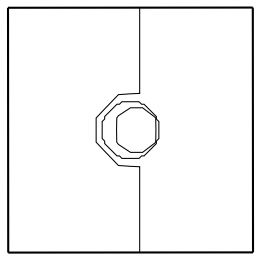

$\mathrm{t}=0.75$

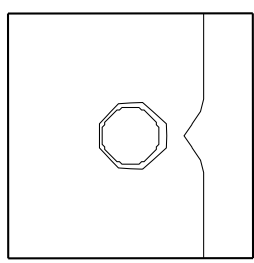

$t=0.9$

Figure 10: Capturing of dislocation lines vs non-local interactions

\subsubsection{Test 4: pinning and cooperative behavior}

Here we illustrate how the non-local interactions between different dislocations can give rise to a cooperative behavior. To this end we consider again a velocity of the form $c_{0}(x)=c_{1}(x)+L$, with $L=1, x=\left(x_{1}, x_{2}\right)$ and

$$
c_{0}(x)= \begin{cases}x_{1}^{2} /\left(\frac{7}{8} x_{2}^{2}+\frac{1}{32}\right)^{2}-1 & \text { if }\left|x_{1}\right| \leq \frac{7}{8} x_{2}^{2}+\frac{1}{32}, \\ 0 & \text { otherwise }\end{cases}
$$

so that the corresponding obstacle is a region with curved boundary which cut in halves the square (see Figure 11). With this choice we both force the whole dislocation line to pass through the obstacle and create self-interactions. Nevertheless Figure 11 shows a single dislocation line $(p=1)$ slowing down rapidly and stopping: its self-force is not enough to overcome the obstacle.

We then consider three lines $(p=3)$, one of them already trapped in the middle of the obstacle. We clarly see in Figure 12 that dislocations help one another periodically. 


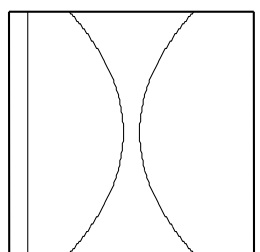

$t=0$

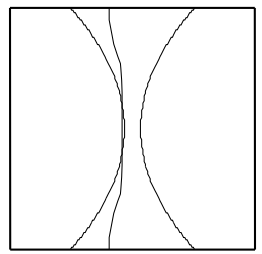

$\mathrm{t}=0.6$

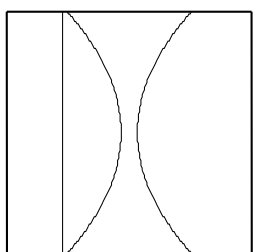

$\mathrm{t}=0.2$

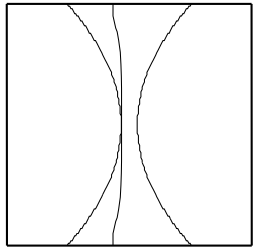

$t=0.9$

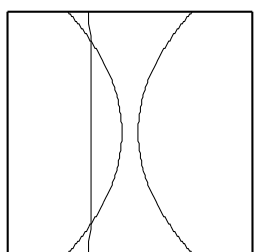

$\mathrm{t}=0.35$

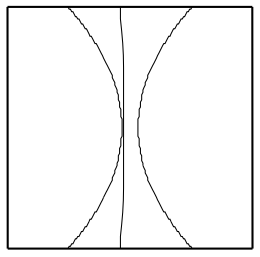

$\mathrm{t}=1.4$

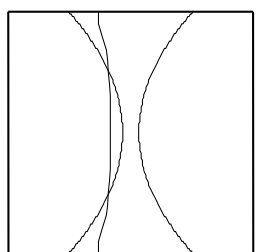

$t=0.45$

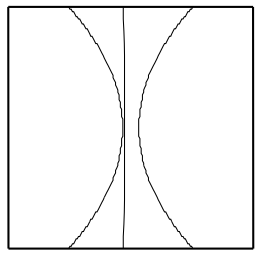

$t=1.9$

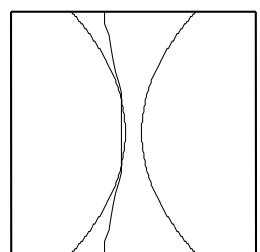

$t=0.5$

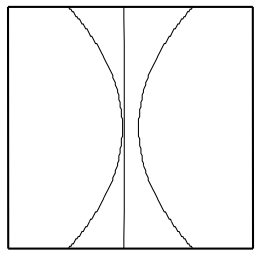

$t=2.4$

Figure 11: Pinning of a single dislocation line: self-interactions are not enough

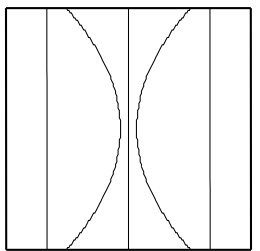

$t=0$

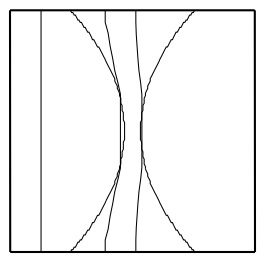

$t=0.4$

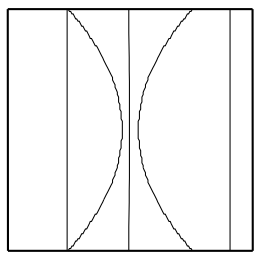

$\mathrm{t}=0.1$

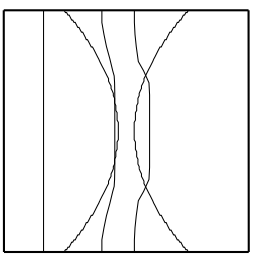

$\mathrm{t}=0.45$

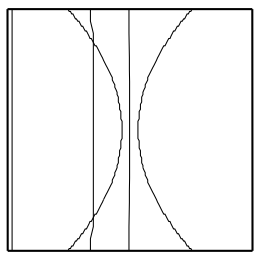

$\mathrm{t}=0.25$

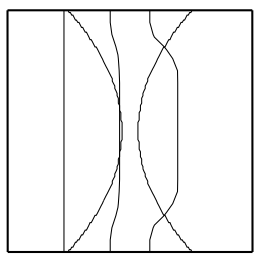

$t=0.55$

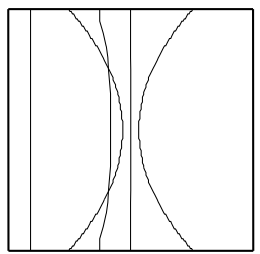

$\mathrm{t}=0.35$

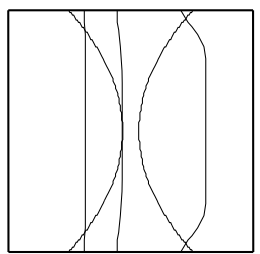

$t=0.68$

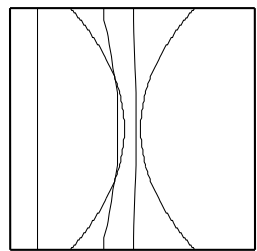

$\mathrm{t}=0.38$

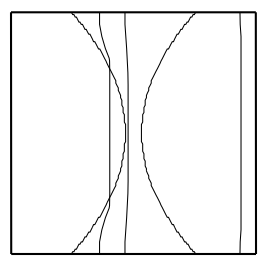

$\mathrm{t}=0.85$

Figure 12: Three dislocation lines in cooperative motion

\section{Aknowledgements}

The authors would like to thank N. Forcadel and A. Ghorbel for fruitful discussions. This work was supported by the contract ANR MICA (2006-2010).

\section{References}

[1] Y. Achdou, F. Camilli, I. Capuzzo Dolcetta, Homogenization of Hamilton-Jacobi equations: numerical methods, Mathematical Models and Methods in Applied Sciences, 18 (7), (2008), 1115-1143.

[2] S. Albert, B. Cockburn, D. A. French, T. E. Peterson, A posteriori error estimates for general numerical methods for Hamilton-Jacobi equations. Part I: the steady state case, Math. Comp., 71, (2001), 49-76. 
[3] S. Albert, B. Cockburn, D. A. French, T. E. Peterson, A posteriori error estimates for general numerical methods for Hamilton-Jacobi equations. Part II: the time-dependent case, Finite volumes for complex applications, III (Porquerolles, 2002), 3-10, Hermes Sci. Publ., Paris, 2002.

[4] O. Alvarez, E. Carlini, R. Monneau, E. Rouy, A convergent scheme for a non local Hamilton Jacobi equation modelling dislocation dynamics, Numerische Mathematik 104 (4), 413-444, 2006.

[5] O. Alvarez, E. Carlini, R. Monneau, E. Rouy, Convergence of a first order scheme for a non local eikonal equation, IMACS Journal "Applied Numerical Mathematics" 56, 1136-1146, (2006).

[6] O. Alvarez, P. Hoch, Y. Le Bouar, R. Monneau, Dislocation dynamics: short time existence and uniqueness of the solution, Archive for Rational Mechanics and Analysis, 181 (3), (2006), 449-504.

[7] G. Barles, P. Cardaliaguet, O. Ley, R. Monneau, General existence results and uniqueness for dislocation equations, SIAM J. Math. Anal. 40 (1), 44-69, (2008).

[8] F. Camilli, I. Capuzzo Dolcetta, D. A. Gomes, Error estimates for the approximation of the effective Hamiltonian, Appl. Math. Optim., 57, (2008), 30-57.

[9] E. Carlini, N. Forcadel, R. Monneau, A Generalized Fast Marching Method for dislocation dynamics, work in progress.

[10] M. G. Crandall, P. L. Lions, Two approximations of solutions of Hamilton-Jacobi equations, Math. Comp., 43, (1984), 1-19.

[11] F. Da Lio, N. Forcadel, R. Monneau, Convergence of a non-local eikonal equation to anisotropic mean curvature motion. Application to dislocations dynamics, Journal of the European Mathematical Society, 10 (4), (2008), 1061-1104.

[12] A. El Hajj and N. Forcadel, A convergent scheme for a non-local coupled system modelling dislocations densities dynamics, Math. Comp. 77,2008, pp 789-812.

[13] A. El Hajj and H. Ibrahim, R. Monneau, Homogenization of dislocation dynamics, accepted to the proceedings of the conference Dislocations 2008, IOP Conferences Series: Materials Science and Engineering.

[14] N. Forcadel, An error estimate for a new scheme for mean curvature motion, SIAM journal on numerical analysis, 46(5), 2008, pp. 2715-2741.

[15] N. Forcadel, C. Imbert, R. Monneau, Homogenization of the dislocation dynamics and of some particle systems with two-body interactions, Discrete and Continuous Dynamical Systems - A, vol. 23 (3) (March 2009), 785 - 826.

[16] A. Ghorbel, P. Hoch, R. Monneau, A numerical study for the homogeneization of one-dimensional models describing the motion of dislocations, accepted to Int. J. of Computing Science and Mathematics.

[17] D. A. Gomes, A. M. Oberman, Computing the effective Hamiltonian using a variational approach, SIAM J. Control Optim., 43 (3), (2004), 792-812.

[18] J. P. Hirth, J. Lothe, Theory of dislocations, Krieger, (1982), Second edition.

[19] C. Imbert, E. Rouy, R. Monneau, Homogenization of first order equations with u/epsilon-periodic Hamiltonians. Part II: application to dislocations dynamics, Communications in Partial Differential Equations 33 (2008), no. 1-3, 479-516.

[20] H. Ishii, Y. Matsumura, Non-local Hamilton-Jacobi equations arising in dislocation dynamics, preprint.

[21] S. Osher, J. A. Sethian, Fronts propagating with curvature-dependent speed: algorithms based on Hamilton-Jacobi, J. Comput. Phys., 79, (1988), 12-49.

[22] M. Rorro, An approximation scheme for the effective Hamiltonian and applications, Appl. Numer. Math, 56, (2006), 1238-1254. 
[23] E. Rouy, A. Tourin, A viscosity solutions approach to shape-from-shading, SIAM J. Numer. Anal., 29 (3), (1992), 867-884.

[24] D. Slepčev, Approximation schemes for propagation of fronts with nonlocal velocities and Neumann boundary conditions, Nonlinear Anal., 52 (2003), pp. 79-115.

[25] J. Qian, Two approximations for effective Hamiltonians arising from homogenization of HamiltonJacobi equations, UCLA, CAM reports, 03-39, 2003. 